ARTICLE

Received 15 Aug 2013 | Accepted 30 Jan 2014 | Published 24 Feb 2014

DOI: $10.1038 /$ ncomms 4356

\title{
Post-fusion structural changes and their roles in exocytosis and endocytosis of dense-core vesicles
}

Hsueh-Cheng Chiang ${ }^{1, \star}$, Wonchul Shin ${ }^{1}{ }^{\star}$, Wei-Dong Zhao ${ }^{1}$, Edaeni Hamid ${ }^{1}$, Jiansong Sheng ${ }^{1}$, Maryna Baydyuk ${ }^{1}$ Peter J. Wen ${ }^{1}$, Albert Jin², Fanny Momboisse ${ }^{1, \dagger} \&$ Ling-Gang $\mathrm{Wu}^{1}$

Vesicle fusion with the plasma membrane generates an $\Omega$-shaped membrane profile. Its pore is thought to dilate until flattening (full-collapse), followed by classical endocytosis to retrieve vesicles. Alternatively, the pore may close (kiss-and-run), but the triggering mechanisms and its endocytic roles remain poorly understood. Here, using confocal and stimulated emission depletion microscopy imaging of dense-core vesicles, we find that fusion-generated $\Omega$-profiles may enlarge or shrink while maintaining vesicular membrane proteins. Closure of fusion-generated $\Omega$-profiles, which produces various sizes of vesicles, is the dominant mechanism mediating rapid and slow endocytosis within $\sim 1-30 \mathrm{~s}$. Strong calcium influx triggers dynamin-mediated closure. Weak calcium influx does not promote closure, but facilitates the merging of $\Omega$-profiles with the plasma membrane via shrinking rather than full-collapse. These results establish a model, termed $\Omega$-exo-endocytosis, in which the fusiongenerated $\Omega$-profile may shrink to merge with the plasma membrane, change in size or change in size then close in response to calcium, which is the main mechanism to retrieve dense-core vesicles.

\footnotetext{
${ }^{1}$ National Institute of Neurological Disorders and Stroke, 35 Convent Drive, Building 35, Room 2B-1012, Bethesda, Maryland 20892, USA. ${ }^{2}$ National Institute of Biomedical Imaging and Bioengineering (NIBIB), Bethesda, Maryland 20892, USA. * These authors contributed equally to this work. ${ }^{\dagger}$ Present address: Centro Interdisciplinario de Neurociencia de Valparaíso, Universidad de Valparaíso, Gran Bretaña 1111, Playa Ancha, Valparaíso 2360102, Chile.

Correspondence and requests for materials should be addressed to L.-G.W. (email: wul@ninds.nih.gov).
} 
E xocytosis, a process that involves the fusion of a vesicle with the plasma membrane to release vesicular contents, is crucial for many biological events, including brain activities and endocrine functions ${ }^{1}$. To maintain exocytosis, fused vesicles are recycled via endocytosis. There are at least two exoendocytosis modes. One involves vesicle full-collapse (FC) into the plasma membrane and spread of vesicular membrane proteins $^{2,3}$, followed by classical endocytosis involving membrane invagination and fission to recycle vesicles ${ }^{4-6}$. The other mode, called kiss-and-run $(\mathrm{KR})^{7,8}$, involves fusion pore opening and closure ${ }^{4-6}$. Although these two modes have been widely accepted, the presumed structural changes have not been observed in live cells at sub-diffraction-limited resolution. Here we used confocal and stimulated emission depletion microscopy $(\mathrm{STED})^{9}$ at $\sim 230 \mathrm{~nm}$ and $90 \mathrm{~nm}$ resolution, respectively, to resolve these hypothesized structural changes in live chromaffin cells containing $\sim 300 \mathrm{~nm}$ dense-core vesicles.

Although decades of studies in chromaffin cells contribute to forming the current FC/KR theory $4,6,10,11$, the physiological role and the trigger mechanism of $\mathrm{KR}$ remain unresolved ${ }^{4,10}$. KR, which was first detected as capacitance flickers in $\sim 0.2 \mathrm{~s}^{12-14}$, was proposed to underlie an entire cell's endocytosis ${ }^{15}$. However, endocytosis recorded from whole-cell capacitance measurements (whole-cell endocytosis) lasts for $\sim 20-30 \mathrm{~s}^{15}$. This long time course is difficult to be accounted for by KR as brief as $\sim 0.2 \mathrm{~s}$. A slower form of $\mathrm{KR}$, termed cavicapture, has been revealed through imaging vesicular membrane proteins ${ }^{15}$. However, cavicapture is not considered the dominant mechanism mediating whole-cell endocytosis ${ }^{15}$. In brief, KR has not been established as the dominant endocytic mode in chromaffin cells. Neither has it been established in other cell types ${ }^{4-6}$. Two factors might contribute to this situation. First, to assess the contribution of $\mathrm{KR}$ to whole-cell endocytosis, it is best to reconstruct the overall endocytosis from individual $\mathrm{KR}$ for comparison with the simultaneously recorded whole-cell endocytosis. This comparison is labour intensive because $\mathrm{KR}$ is not trivial to record ${ }^{13,15}$. Second, the stimulation condition and the mechanism that predominantly triggers $\mathrm{KR}$ remain elusive ${ }^{4-6,16}$. KR is proposed to be triggered by low calcium $^{17-19}$. Paradoxically, higher calcium influx speeds up wholecell endocytosis in chromaffin cells ${ }^{20,21}$ and other cell types ${ }^{2-24}$.

In the present work, we develop an imaging technique with high spatial and temporal resolution to visualize structural changes of fusion-generated $\Omega$-shaped membrane profile while simultaneously monitoring whole-cell endocytosis with capacitance measurements in chromaffin cells. We find that $\Omega$-profile does not dilate as predicted by the FC model or simply close its pore as predicted by the KR model, but dynamically changes in seven modes. These results establish a model that redefines FC and KR fusion as part of a larger spectrum of structural changes, with varied triggers and physiological roles. We suggest to redefine KR as rapid or slow closure of $\Omega$-shaped profile, during which this profile may change in size before closure, resulting in various sizes of vesicles. Pore closure is mediated by dynamin and triggered by large calcium influx. It is the predominant mechanism mediating whole-cell rapid and slow endocytosis lasting for $\sim 1-30 \mathrm{~s}$. It may also mediate bulk endocytosis, a form of endocytosis that generates large vesicles ${ }^{5,6}$. Low calcium influx facilitates shrinking of the fusion-generated $\Omega$-shaped profile, leading to the merge of the fused vesicle with the plasma membrane. These results call for substantial modification of the classical FC and KR model.

\section{Results}

Imaging $\Omega$-profile at the instant of fusion. To label $\Omega$-shaped membrane profiles and assess its structural changes, primary cultured bovine chromaffin cells were bathed with membraneimpermeable fluorescent dyes (Fig. 1a) ${ }^{25}$. With Alexa Fluor 647 $(\mathrm{A} 647,30 \mu \mathrm{M})$ in the bath, cells were voltage clamped while imaged confocally every $5-15 \mathrm{~ms}$ at the cell-bottom (applies if not mentioned otherwise, Fig. 1a). A $1 \mathrm{~s}$ depolarization (depol $\mathrm{l}_{1 \mathrm{~s}}$, from $-80 \mathrm{mV}$ to $+10 \mathrm{mV}$, if not mentioned otherwise) induced a calcium current (ICa) of $309 \pm 34$ pA (mean \pm s.e.m., $n=60$ ), a capacitance jump $(\Delta \mathrm{Cm})$ of $365 \pm 33 \mathrm{fF}(n=60)$ and $10.6 \pm 1.2$ fluorescent A647 spots in $\sim 70-160 \mu \mathrm{m}^{2}$ of the foot area of the cell $(n=60$ cells, Fig. $1 \mathrm{~b})$. This stimulation protocol was used because it consistently induces endocytosis (Fig. 1b) ${ }^{15,26}$.

Three sets of evidence suggest that A647 spots reflect vesicle fusion, owing to A647 diffusion from the bath to open vesicles. First, most spots occurred during and within $1 \mathrm{~s}$ after depolarization (Fig. 1c, upper, 60 cells, 636 spots in total). This time course (Fig. 1c, upper) was parallel to the immediate capacitance jump induced by depolarization, which reflects exocytosis (Fig. 1c, lower). The spot number per cell was also proportional to the $\Delta \mathrm{Cm}$ (Fig. 1d). Second, analogous to calcium-triggered exocytosis, removing extracellular calcium abolished both spot occurrence and $\Delta \mathrm{Cm}(n=10$ cells, $P<0.01$, Supplementary Fig. 1). Third, in cells expressing neuropeptide Y-EGFP (NPYEGFP, granule lumen cargo), $97 \%(=175 / 181)$ of the NPY-EGFP release events were accompanied with A647 spots (Fig. 1e, arrows). The remaining $3 \%$ were likely too small or fast to be resolved. $58 \pm 5 \%$ of A647 spots ( $n=350$ spots, 23 cells) overlapped with NPY-EGFP-positive granules and their release (Fig. 1e). The percentage of overlap increased linearly towards $100 \%$ as the NPY-EGFP-positive granule density increased (Fig. 1f). Thus, A647 spots that did not overlap with NPYEGFP-positive granules were due to fusion of NPY-EGFPnegative granules.

Four sets of evidence suggest that the A647 spot at the onset reflects an $\Omega$-profile at the fusion instant. First, under the confocal microscope, the full-width-half-maximum $\left(W_{\mathrm{H}}\right)$ of NPY-EGFPpositive granule $(475 \pm 13 \mathrm{~nm}, n=48$ granules) was similar to the overlapping A647 spot's $W_{\mathrm{H}}$ at the onset $(490 \pm 13 \mathrm{~nm}, n=48$ spots, $P=0.15, \quad t$-test; Fig. $2 \mathrm{a})$. STED imaging $(\sim 90 \mathrm{~nm}$ resolution) confirmed that the $W_{\mathrm{H}}$ of NPY-EGFP-positive vesicle ( $332 \pm 5 \mathrm{~nm}, n=278$ granules, 7 cells) was similar to the $W_{\mathrm{H}}$ of spots $\left(369 \pm 10 \mathrm{~nm}, n=101\right.$ spots, 31 cells) induced by depol ${ }_{1 \mathrm{~s}}$ in different cells bathed with Alexa Fluor 488 (A488, 30-60 $\mu \mathrm{M}$, Fig. 2b). Note that A488 or EGFP, but not A647 can be used in our STED microscope. The spot $W_{\mathrm{H}}$ measured with STED microscopy was close to electron microscopic measurements $(\sim 300 \mathrm{~nm})^{13}$, supporting the idea that fused vesicles do not collapse.

Second, to visualize these structures, we conducted STED imaging at the cell-centre ( $>2 \mu \mathrm{m}$ above bottom), where the plasma membrane was approximately perpendicular to the coverslip (Fig. 1a). Depol 1 induced A488 spots only adjacent to the cell membrane (Fig. $2 c, n=45$ ). These 'side' images showed $\Omega$-like profiles with a pore beyond our resolution (Fig. 2c).

To quantify the $\Omega$-profile, we simulated an $\Omega$-profile with a diameter of $300 \mathrm{~nm}$ and a pore $<100 \mathrm{~nm}$ (Fig. 2d, see Supplementary Fig. 2 for derivation). With a dye outside the cell membrane, line profiles through the simulated $\Omega$-profile centre showed a dip right before reaching the membrane (Fig. $2 \mathrm{~d}$, arrow). As expected for an $\Omega$-profile, the dip for a $45^{\circ}$ line was larger and wider than a horizontal line (Fig. 2d). These features were not observed in a simulated 'FC' profile with an opening that is the same or larger than the vesicle diameter (Fig. 2e, Supplementary Fig. 3).

The image and line features of the simulated $\Omega$-profile were also observed in STED side images of A488 spots (Fig. 2c, arrow), confirming that A488 spots were $\Omega$-profiles. After depolarization, 
a

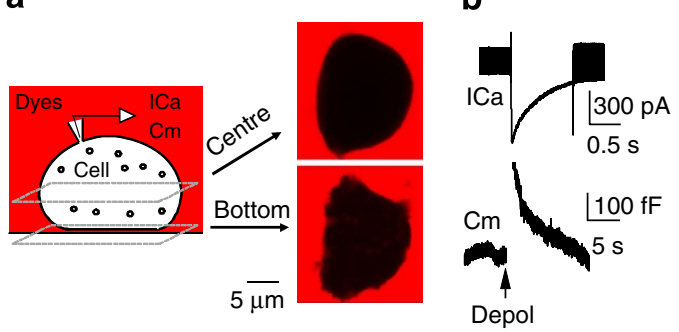

d

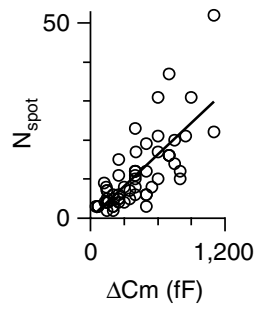

e

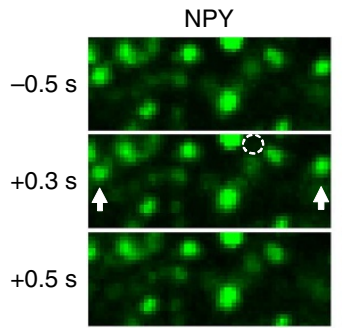

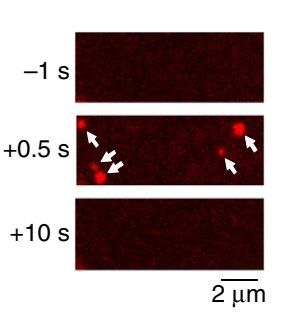

C

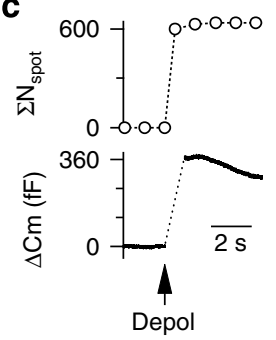

f

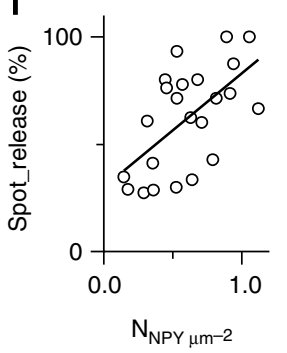

Figure 1 | Imaging granule fusion in chromaffin cells. (a) Left: Schematic drawing showing whole-cell recording of ICa and capacitance (Cm), and imaging at the cell-bottom or centre with a fluorescent dye (red) in the bath. Right: Confocal images at a real cell's bottom (lower) or centre (upper) with A647 $(30 \mu \mathrm{M})$ in the bath. The dark area at the cell-bottom represents a thin layer of A647 solution between the cell-bottom and the coverslip. (b) Left: Sampled $\mathrm{ICa}$ (upper) and $\mathrm{Cm}$ (lower) induced by depol ${ }_{1 \mathrm{~s}}$ (arrow). Right: A647 confocal/cell-bottom images at $1 \mathrm{~s}$ before, during $(0.5 \mathrm{~s})$ and $10 \mathrm{~s}$ after depol $1 \mathrm{~s}$ (same cell as from left). Arrows indicate $A 647$ spots. (c) The accumulated number of $A 647$ spots ( $\Sigma N_{\text {spot, }}$ upper) plotted versus the time at which the spots occurred in 60 cells subjected to depol $_{1 \mathrm{~s}}$ (arrow). The corresponding mean $\mathrm{Cm}$ change is also plotted (lower). (d) The A647 spot number $\left(N_{\text {spot }}\right)$ plotted versus $\mathrm{Cm}$ from the same cell $(n=60$, each circle represents one cell). Data were fitted with a linear regression line (correlation coefficient: 0.71). (e) Concurrent imaging of NPY-EGFP (green) and A647 (red) at $0.5 \mathrm{~s}$ before (upper), and $0.3 \mathrm{~s}$ (middle) and $0.5 \mathrm{~s}$ (lower) during depol ${ }_{1 \mathrm{~s}}$. Arrows indicate NPY-EGFP release (green spots disappeared at $0.5 \mathrm{~s}$ ) coincident with A647 spots. Circles indicate an A647 spot without an overlapping NPY-EGFP spot. (f) The percentage of A647 spots that are co-localized with NPY-EGFP release (spot_release) plotted versus the number of NPY-EGFP granules per $\mu \mathrm{m}^{2}$ at the cell-bottom $\left(N_{\mathrm{NPY}} \mu \mathrm{m}^{-2}\right)$. Each circle represents one cell $(n=23$ cells; cells with $>5$ A647 spots were used).

we did not observe spots that resembled the simulated collapselike images and line features (see Methods for criteria of side imaging). However, we did observe some resting membrane curvatures (Fig. 2f) resembling the simulated 'collapse' image and line features (Fig. 2e). Thus, the lack of 'collapse' profiles after depolarization was not due to limited spatial resolution.

Third, the $\Omega$-profile appeared at the spot onset (within $36 \mathrm{ms,}$ our time resolution, 45 spots, see also Figs 3-5). This rapid appearance, together with the overlap between the spot appearance and NPY-EGFP release (Fig. 1e,f), suggests that the $\Omega$-profile is due to fusion, but not slow, endocytic membrane invagination.

Fourth, some spots may close their pore without detectable structural changes (for example, $\Omega$-close fusion, described later), confirming that the $\Omega$-profile's pore is too small to be resolved.

Seven modes of $\boldsymbol{\Omega}$-profile change. To determine how $\Omega$-shaped membrane profiles change in size and pore opening or closing status, we performed confocal imaging (every 5-15 ms) at the cell-bottom with A647 and A488 in the bath being excited strongly and weakly, respectively (A647/A488 experiments, reasons explained later). We found that the structures may change in seven ways described below (636 spots, 60 cells), which reflect different size changes of the $\Omega$-profile (no change, enlarge, shrink partially or completely) and the pore status (opened or closed). These patterns were confirmed with STED imaging at the cellbottom (STED/cell-bottom, every $26 \mathrm{~ms}$, strong excitation) and cell-centre (STED/cell-centre, every $36 \mathrm{~ms}$, strong excitation).

In $11 \%$ of the spots (73/636 spots) in confocal/A647/A488 experiments, A647 and A488 fluorescence intensity $\left(F_{647}, F_{488}\right)$ peaked mostly rapidly $(<100 \mathrm{~ms})$ and stayed unchanged $(<25 \%)$ for $30 \mathrm{~s}$ (the end of our image recording), during which A647 or A488 spot's $W_{\mathrm{H}}$ remained stable (A647: $485 \pm 8 \mathrm{~nm}$ at onset, $474 \pm 7 \mathrm{~nm} 30 \mathrm{~s}$ later, $n=73$ spots; $P=0.56$, $t$-test; Fig. 3a, Supplementary Movie 1). Similar results were confirmed with STED/cell-bottom imaging: $W_{\mathrm{H}}$ was $380 \pm 35 \mathrm{~nm}$ at the onset, and was $400 \pm 40 \mathrm{~nm} 6-8 \mathrm{~s}$ later $(n=5 ; P=0.74$; Fig. $3 \mathrm{~b})$. STED data beyond $\sim 8-10 \mathrm{~s}$ after stimulation were discarded, because the whole-cell configuration was often lost due to strong STED depletion laser. STED/cell-centre imaging showed an $\Omega$-profile with a dip in line profiles, which remained stable for 6-8 s $(n=6$, Fig. 3c). This pattern is termed $\Omega$-stay with an open pore. The evidence supporting an open pore is explained below.

In $33 \%$ of the spots in A647/A488 experiments, $F_{647}$ and $F_{488}$ peaked simultaneously $(<70 \mathrm{~ms})$. Subsequently, $F_{647}$ remained unchanged for $0.3-30 \mathrm{~s}$, then decayed monoexponentially to baseline with a $\tau$ of $2.9 \pm 0.1 \mathrm{~s}$, whereas $F_{488}$ remained unchanged ( $n=210$ spots, Fig. 3d,e; Supplementary Movie 2). The A647 or A488 spot's $W_{\mathrm{H}}$ also remained stable: A647 spot's $W_{\mathrm{H}}$ was $474 \pm 5 \mathrm{~nm}$ at the $F_{647}$ peak, and was $462 \pm 4 \mathrm{~nm}$ when $F_{647}$ decayed to $25 \pm 1 \%$ of the peak $(n=210, P=0.18$, $t$-test; Fig. 3d,e). STED/cell-bottom imaging confirmed this result: $W_{\mathrm{H}}$ was $371 \pm 17 \mathrm{~nm}$ at the fluorescence $\left(F_{\mathrm{STED}}\right)$ peak, and was $351 \pm 16 \mathrm{~nm}$ at $20-30 \%$ of $F_{\text {STED }}$ peak $(n=27, P=0.43$, Fig. $3 f)$. This pattern, termed here as $\Omega$-close, reflects $\Omega$-profile pore closure resembling $\mathrm{KR}^{7,8}$, except that closing time can be long. The following two sets of evidence support this conclusion.

First, when $F_{647}$ (strong excitation) but not $F_{488}$ decayed, removing A647 excitation halted $F_{647}$ decay, and resuming excitation recommenced $F_{647}$ decay $(n=11$ spots, Supplementary Fig. 4a). After $F_{647}$ decay, increasing A488 excitation increased 
a

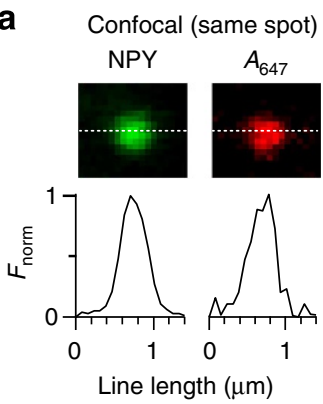

d
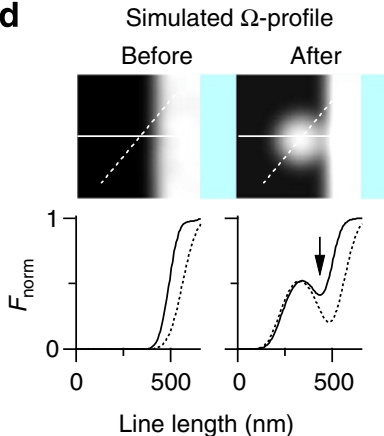

b

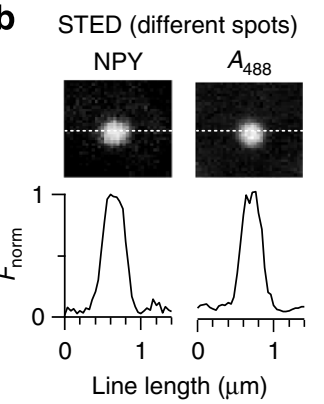

e

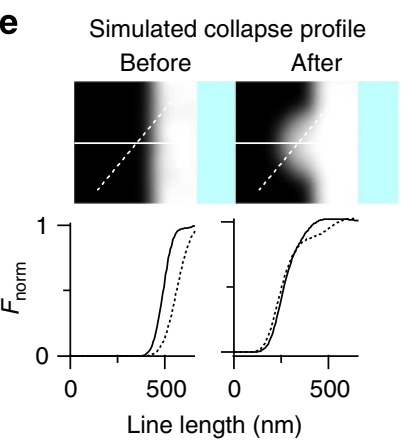

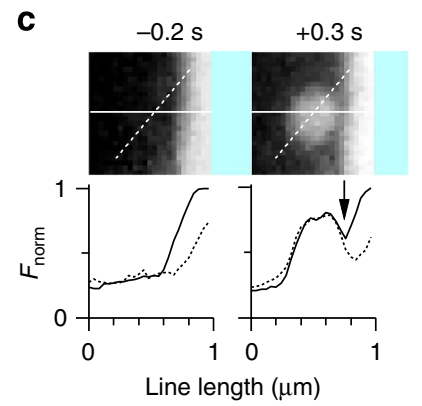

Collapse-like profile
Example $1 \quad$ Example 2

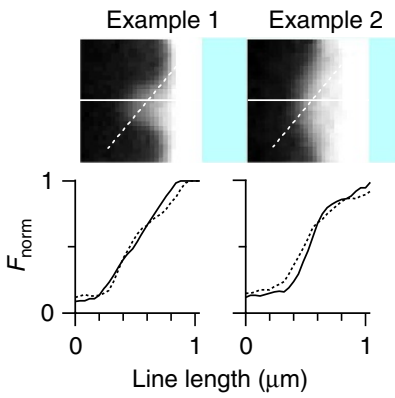

Figure 2 | Resolving the $\mathbf{\Omega}$-profile at the fusion instant. (a) Confocal images of a NPY-EGFP-positive granule before release and the A647 spot at the spot onset at the same location (upper). Normalized fluorescence intensity profiles ( $F_{\text {norm }}$ ) from dotted lines are also shown (lower, applies to b). (b) STED images (upper) of a NPY-EGFP granule and an A488 spot at the spot onset (from different cells). Line profiles are also plotted (lower). (c) STED/cell centre images at $0.2 \mathrm{~s}$ before (left) and $0.3 \mathrm{~s}$ (right) during depol ${ }_{1 \mathrm{~s}}$ (upper). $F_{\text {norm }}$ are also shown for two lines across the spot centre, one perpendicular to the plasma membrane, the other $45^{\circ}$ apart (applies to $\mathbf{d}-\mathbf{f}$ ). The arrow indicates the typical feature of the $\Omega$-profile: a dip in the line profile that is wider and larger for the $45^{\circ}$ line. Bright fluorescence in the right side of each image represents extracellular A488, whereas dim fluorescence in the left side of each image means the intracellular compartment with no A488 (applies to all plots at the STED per cell-centre setting). (d, e) Simulation showing side images and line profiles (solid and dotted) before (left) and after (right) the appearance of an $\Omega$-profile (d, pore size: $50 \mathrm{~nm}$, vesicle size: $300 \mathrm{~nm}$ ) or a collapsed profile (e). The arrow in d indicates the typical feature of the $\Omega$-profile: a dip in the line profile which is wider and larger for the $45^{\circ}$ line. Images are taken from Supplementary Figs 2f,g and 3c. Simulation methods are described in Supplementary Figs 2,3. (f) Two STED/cell-centre images and line profiles (left, right) that resemble the presumed collapsed profile. Images were obtained in resting conditions.

$F_{488}$, but followed by a monoexponential decay $(n=10$ spots, Supplementary Fig. 4 b). Thus, strong excitation decreased $F_{647}$ (or $F_{488}$ ). However, strong excitation alone was insufficient to cause $F_{647}$ decay, because $F_{647}$ may remain stable (for example, Fig. 3a) or decay at different onsets (for example, Fig. 3d,e). Thus, $F_{647}$ decay must reflect pore closure, which prevents exchange of bleached dye (caused by strong excitation) with fluorescent dye in the bath. In contrast, a stable $F_{647}(\Omega$-stay) reflects an open pore (Fig. 3a-c).

Second, as described later, endocytosis reconstructed from $\Omega$ close and other 'close' modes matched whole-cell endocytosis. Moreover, block of whole-cell endocytosis by prolonging wholecell dialysis or inhibition of dynamin abolished 'close' fusion. Thus, 'close' modes reflect endocytosis.

In $8 \%$ of the spots in A647/A488 experiments, $F_{647}$ and $F_{488}$ increased in parallel in two phases, initially within $\sim 300 \mathrm{~ms}$ and subsequently in $\sim 1-20 \mathrm{~s}$ (Fig. $4 \mathrm{a}$ ). $W_{\mathrm{H}}$ at the onset was $486 \pm 8 \mathrm{~nm}(n=52)$, similar to $\Omega$-stay $(485 \pm 8 \mathrm{~nm}, n=73$, $P=0.78$, $t$-test $)$ or $\Omega$-close $(474 \pm 5 \mathrm{~nm}, n=210, P=0.14)$. It increased to $600 \pm 12 \mathrm{~nm}$ at the fluorescence peak $(n=52$, $P<0.001$, Fig. 4a). STED/cell-bottom imaging showed similar patterns: $W_{\mathrm{H}}$ at the onset was $399 \pm 13 \mathrm{~nm}(n=8)$, similar to that of $\Omega$-stay $(380 \pm 435 \mathrm{~nm}, \quad n=5, \quad P=0.60) \quad$ or $\Omega$-close $(371 \pm 17 \mathrm{~nm}, n=27, P=0.42)$, but increased to $508 \pm 26 \mathrm{~nm}$ $(n=8)$ at the peak $(P<0.01)$. STED/cell-centre imaging revealed an $\Omega$-profile (with a dip in line profiles) and its enlargement towards the cytosol ( $n=6$ spots, Fig. 4b, Supplementary Movie 3 ). This mode is termed $\Omega$-enlarge-stay. The slow time course is not due to slow diffusion of the dye into the vesicle, but to the slow size increase, because the initial spot $W_{\mathrm{H}}$ was similar to $\Omega$-stay or $\Omega$-close, and the dye diffuses to a granule in milliseconds 27 .

About 2\% of the spots in A647/A488 experiments showed initial changes similar to $\Omega$-enlarge-stay, but followed by $F_{647}$ decay to baseline with a $\tau(2.8 \pm 0.2 \mathrm{~s}, n=15)$ similar to that of $\Omega$-close while $F_{488}$ remained unchanged (Fig. 4c). This pattern reflected $\Omega$-profile enlargement and closure, termed $\Omega$-enlarge-close.

In $13 \%$ of the spots in A647/A488 experiments, $F_{647}$ and $F_{488}$ peaked rapidly $(<50 \mathrm{~ms})$ and subsequently decreased in parallel by $50-89 \%$ with a $\tau$ of $273 \pm 41 \mathrm{~ms} \quad(n=84)$, then stayed unchanged for $30 \mathrm{~s}$ (Fig. $5 \mathrm{a}$ ). $W_{\mathrm{H}}$ at the onset was $505 \pm 8 \mathrm{~nm}$, similar to that of $\Omega$-stay, but decreased to $359 \pm 6 \mathrm{~nm}$ at the steady-state $(n=84, P<0.001, t$-test, Fig. $5 a)$. STED/cell-bottom imaging confirmed the spot shrinkage: $W_{\mathrm{H}}$ decreased from $379 \pm 26$ to $237 \pm 18 \mathrm{~nm} \quad(n=22, P<0.01, t$-test, Fig. $5 \mathrm{~b}$, Supplementary Movie 4), and the STED fluorescence intensity $\left(F_{\mathrm{STED}}\right)$ in the spot's outer ring decayed faster to a lower value near baseline than the spot's centre ( $n=22$, for example, Fig. $5 b)$. Consistently, STED/cell-centre imaging showed that the $\Omega$-profile (with a dip in line profiles) shrank towards the plasma membrane without vesicle budding off, then maintained the $\Omega$-profile ( $n=12$ spots, Fig. 5 c), termed $\Omega$-shrink-stay.

In $14 \%$ of the spots in A647/A488 experiments, $F_{647}$ and $F_{488}$ showed initial patterns similar to $\Omega$-shrink-stay, except that after a variable delay $(0.3-30 s)$ in the 'stay' phase, $F_{647}$ decayed to 
a
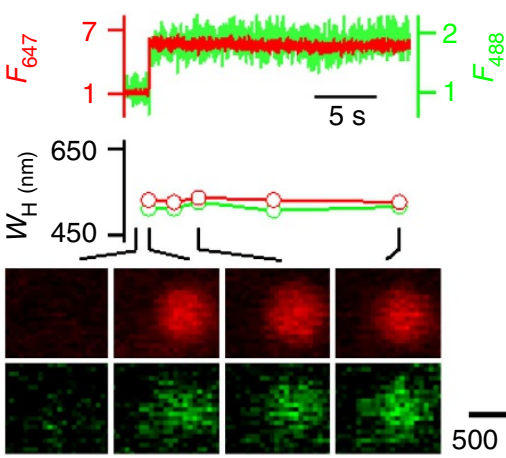

$500 \mathrm{~nm}$ b
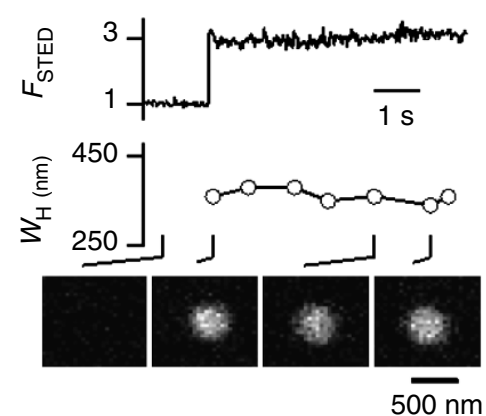

C

$\Omega$-stay (side)
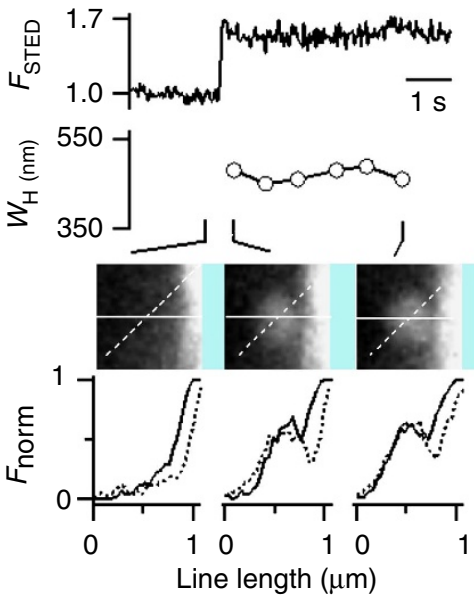

f

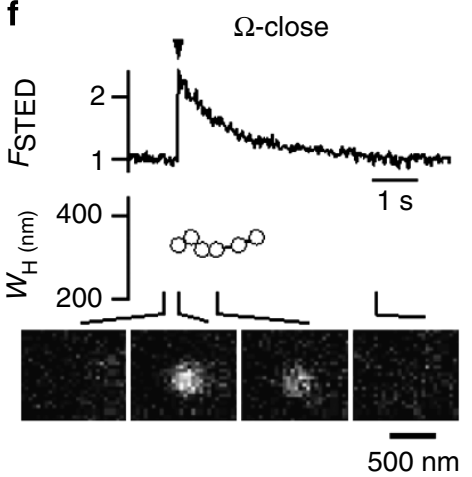

$500 \mathrm{~nm}$ d

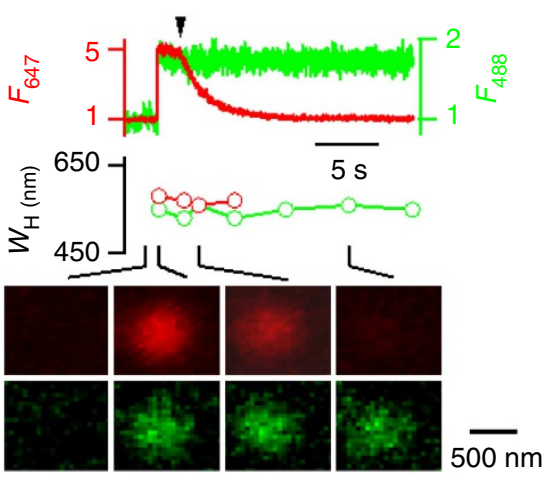

$\mathbf{e}$
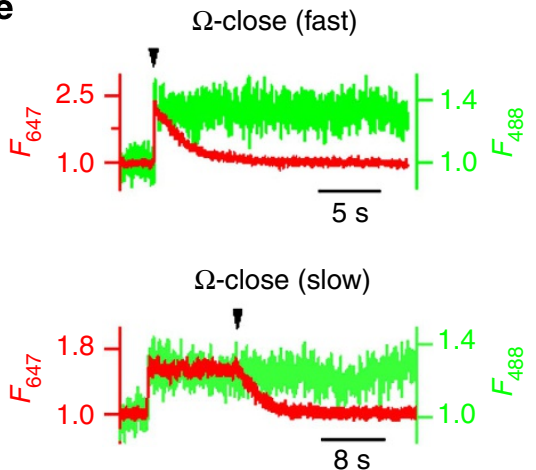

Figure 3 | $\boldsymbol{\Omega}$-stay and $\boldsymbol{\Omega}$-close fusion. (a-c), $\Omega$-stay. (a) $F_{647}$ (red), $F_{488}$ (green), $W_{H}$ and sampled images (average of 4 ) at times indicated (lines) are plotted versus time for a spot at the confocalA647/A488 setting (cell-bottom). $F_{647}$ and $F_{488}$ were normalized to the mean value before spot appeared (applies to all plots of $F_{647}, F_{488}$, and $F_{\text {STED }}$ ). Images were collected every $15 \mathrm{~ms}$. (b) $F_{\text {STED }}$ (STED fluorescence intensity), $W_{H}$, and sampled images

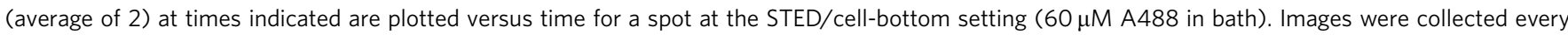
$26 \mathrm{~ms}$. (c) $F_{\text {STED }}, W_{H}$, sampled images (average of 8 , side images of the $\Omega$-profile) and their line profiles (normalized to peak, $F_{\text {norm }}$ ) are plotted versus time for a spot at the STED/cell-centre setting. Images were collected every $36 \mathrm{~ms}$. $W_{H}$ was measured from the profile of a vertical line (not shown, parallel to cell membrane) across the spot centre. Solid and dotted line profiles correspond to solid and dotted lines, respectively. The arrangements in $\mathbf{a}$, $\mathbf{b}$ and $\mathbf{c}$ apply to all plots in Figs 3-6 at confocal/A647/A488, STED/cell-bottom, and STED/cell-centre setting, respectively. (d-f) $\Omega$-close at confocal/A647/A488 (d, e) and STED/cell-bottom setting (f). Arrows indicate pore closure (apply to 'close' fusion in Figs 3-6). E shows two spots (upper, lower) with different pore closing time $\left(W_{H}\right.$ and sampled images not shown).

a
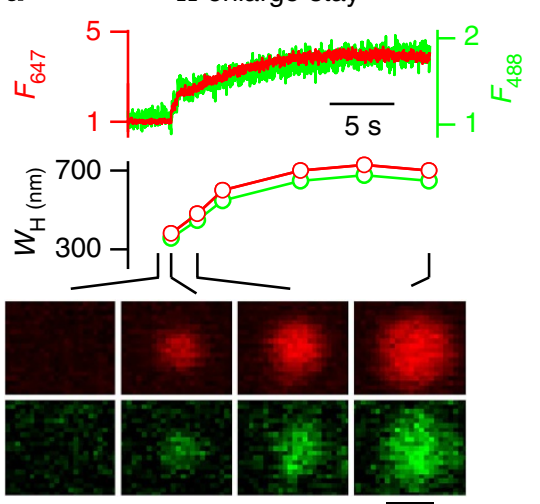

$500 \mathrm{~nm}$ b
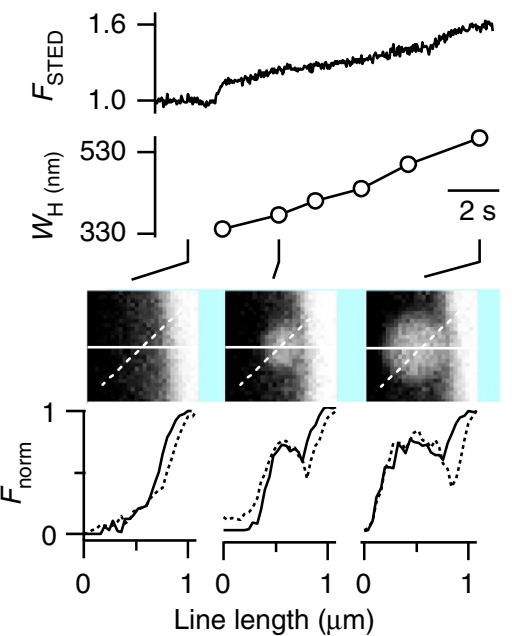

C
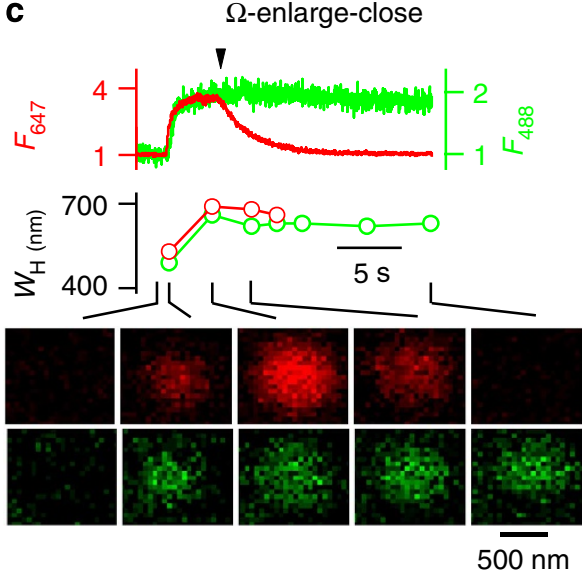

Figure 4 | $\mathbf{\Omega}$-enlarge-stay and $\Omega$-enlarge-close. (a,b) $\Omega$-enlarge-stay at confocal/A647/A488 (a) and STED/cell-centre setting (b). (c) $\Omega$-enlarge-close at confocal/A647/A488 setting. 

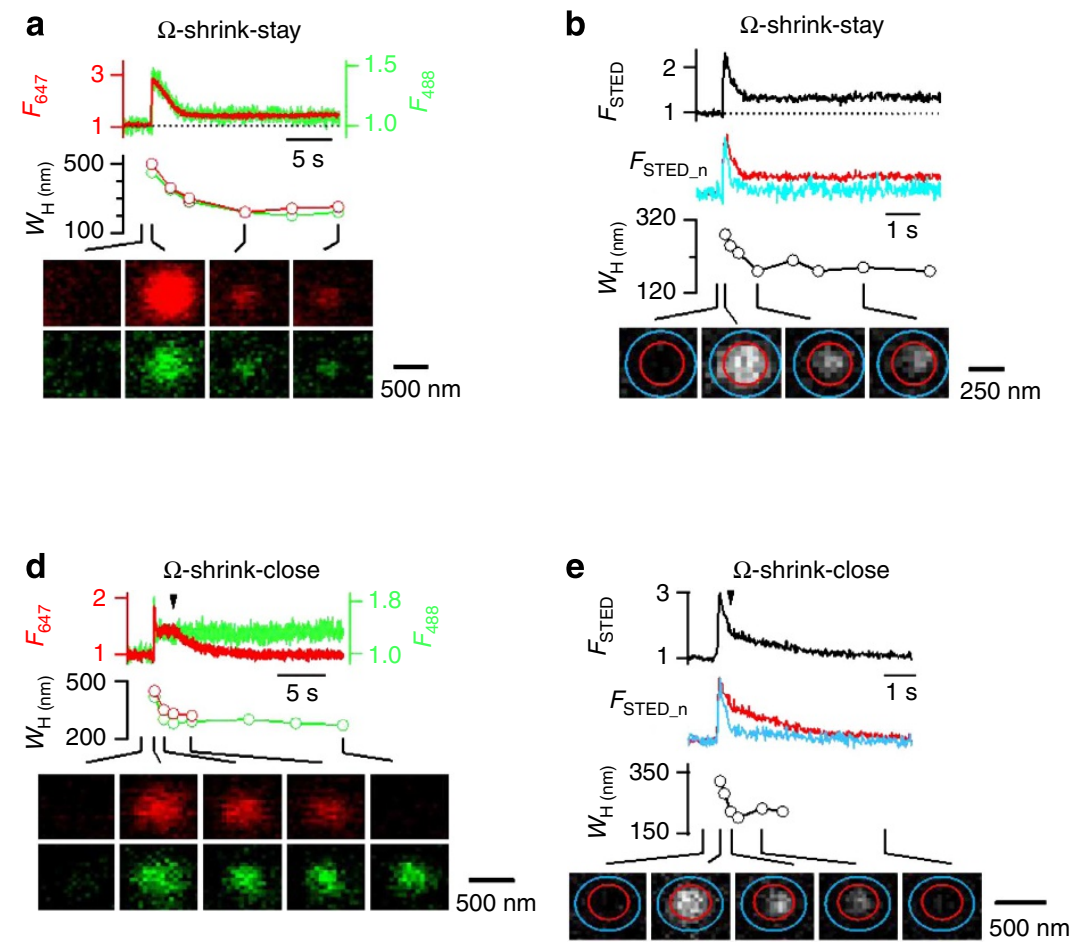

C

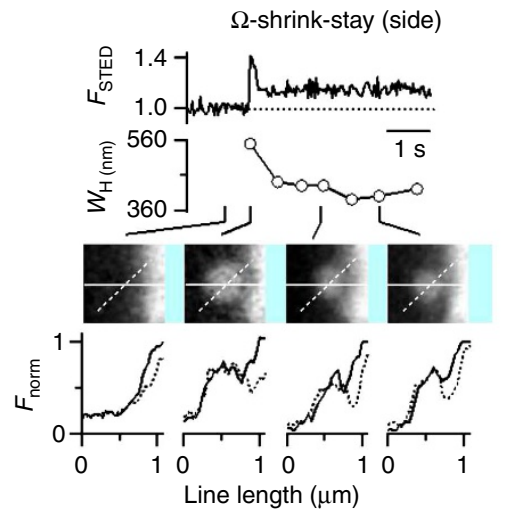

$\mathbf{f}$

$\Omega$-shrink-close (side)
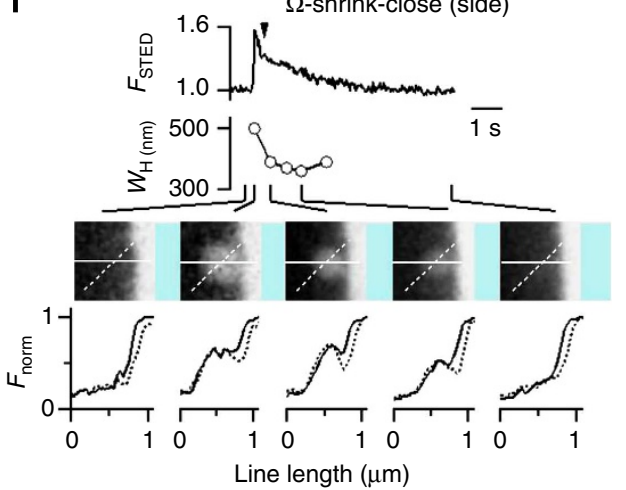

Figure 5 | $\mathbf{\Omega}$-shrink-stay and $\mathbf{\Omega}$-shrink-close. (a-c) $\boldsymbol{\Omega}$-shrink-stay at confocal/A647/A488 (a), STED/cell-bottom (b) and STED/cell-centre setting (c). In $\mathbf{a}$, the scale was set to see dim red images, but partly saturate the brightest red image. In $\mathbf{b}, F_{\text {STED }}$ (peak normalized, $F_{\text {STED_n }}$ ) in the inner circle (red) and the outer ring (between red and blue circles, blue) are also plotted, showing faster decay of blue trace and thus the spot shrinkage. (c) Left two images, average of two single images; right two images, average of eight single images. (d-f) $\Omega$-shrink-close at confocal/A647/A488 (d), STED/cellbottom (e) and STED/cell-centre setting (f). In $\mathbf{e}, F_{\text {STED_n }}$ in the inner circle (red) and the outer ring (between red and blue circles, blue) are also plotted to show spot shrinkage. (f) Left two images, average of two single images; right three images, average of eight single images.

baseline with a $\tau(2.8 \pm 0.1 \mathrm{~s}, n=87)$ similar to that of $\Omega$-close while $F_{488}$ remained unchanged (Fig. $5 \mathrm{~d}$ ). The spot $W_{\mathrm{H}}$ was $509 \pm 7 \mathrm{~nm}$ at the onset, decreased to $418 \pm 5 \mathrm{~nm}(n=87$, $P<0.001, t$-test) at the temporary 'stay' phase, then remained unchanged as $F_{647}$ decayed ( $409 \pm 6 \mathrm{~nm}$ at $\sim 30 \%$ of the 'stay' amplitude, $n=87, P=0.51$, $t$-test, Fig. $5 \mathrm{~d}$ ). STED/cell-bottom imaging showed similar pattern: $W_{\mathrm{H}}$ decreased from $356 \pm 18 \mathrm{~nm}$ at the onset to $244 \pm 12 \mathrm{~nm}(n=20, P<0.001, t$-test $)$ at the temporary 'stay' phase, then remained stable as $F_{\text {STED }}$ decayed to baseline $(254 \pm 13 \mathrm{~nm}$ at $\sim 30 \%$ of amplitude at the transition phase, $n=20, P=0.47$, $t$-test; Fig. $5 \mathrm{e}) . F_{\text {STED }}$ in the spot's outer ring decayed faster to a lower value than the spot's centre in the initial shrinking phase, indicating $\Omega$-profile shrinkage $(n=20$, for example, Fig. 5e). Consistently, STED/cell-centre imaging showed that the $\Omega$-profile (and the dip in line profiles) initially shrank towards the plasma membrane, then its size stayed unchanged while $F_{\text {STED }}$ decayed to baseline ( $n=13$ spots, Fig. $\left.5 f\right)$. These results reflected the $\Omega$-profile shrinkage and closure, termed $\Omega$-shrink-close.

In $18 \%$ of the spots in A647/A488 experiments, $F_{647}$ and $F_{488}$ peaked rapidly $(<50 \mathrm{~ms})$, then decreased in parallel to baseline with a $\tau(1.09 \pm 0.11 \mathrm{~s}, n=115$, Fig. 6a,b) faster than the dye bleaching $\tau$ during $\Omega$-close $(2.9 \pm 0.1 \mathrm{~s}, n=210, P<0.001$, $t$-test). The decay $\tau$ was mostly less than $1.5 \mathrm{~s}$, and sometimes only $15-50 \mathrm{~ms}$ (Fig. 6b). The $W_{\mathrm{H}}$ decreased from $504 \pm 12 \mathrm{~nm}$ at the onset to $365 \pm 10 \mathrm{~nm}(n=115)$ at $23 \pm 1 \%$ of the peak $F_{647}$ (for example, Fig. 6a), beyond which $W_{\mathrm{H}}$ was too dim to measure. STED/cell-bottom imaging confirmed this pattern: $W_{\mathrm{H}}$ decreased from $362 \pm 33 \mathrm{~nm}$ at the onset to $225 \pm 27 \mathrm{~nm}$ at $16 \pm 2 \%$ of the $F_{\text {STED }}$ peak $(n=19, P<0.01, t$-test; Fig. $6 c$, Supplementary Movie $5) . F_{\text {STED }}$ in the spot's outer ring decayed faster than the centre ( $n=19$, Fig. 6c), confirming $\Omega$-profile shrinkage. STED/cellcentre imaging showed shrinkage of the $\Omega$-profile (and the dip in line profile) without vesicle budding off $(n=8$, Fig. $6 \mathrm{~d}$, Supplementary Movie 6), termed $\Omega$-shrink.

In summary, we described seven ways in which an $\Omega$-shaped membrane profile can change, including three close modes $(\Omega$ close, $\Omega$-enlarge-close, $\Omega$-shrink-close), three stay modes $(\Omega$-stay, $\Omega$-enlarge-stay, $\Omega$-shrink-stay) and $\Omega$-shrink (Fig. 7a). Since we limited imaging to $30 \mathrm{~s}$, pore closure for stay modes beyond $30 \mathrm{~s}$ is possible. In addition, we observed occasional events not following these typical patterns. Atypical changes included pore closure and reopening, reflected as $F_{647}$ bleaching (pore closure) and sudden increase to the original level (pore reopening) while $F_{488}$ remained unchanged; $\Omega$-stay followed by $\Omega$-shrink or $\Omega$-shrinkstay, reflected as stable $F_{647}$ value followed by parallel decrease of $F_{647}$ and $F_{488}$; and $\Omega$-enlarge-stay followed by $\Omega$-shrink-stay, reflected as parallel increase and then decrease of both $F_{647}$ and $F_{488}$. These events reflect the continuous nature and flexibility of post-fusion structural changes.

No FC fusion. We did not observe FC fusion (Fig. 7b). The predicted structural change of FC would be spot enlargement while dimming at the cell-bottom, and the collapse of the $\Omega$-profile (Fig. 2e,f) at side images. Neither of these structural 
a

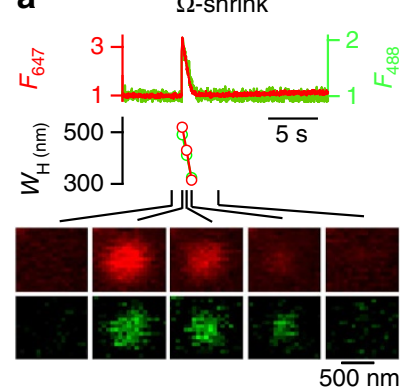

b

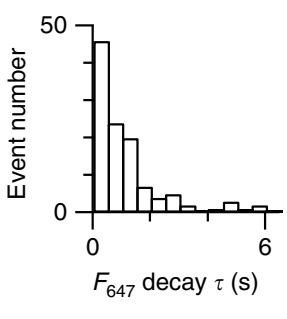

c

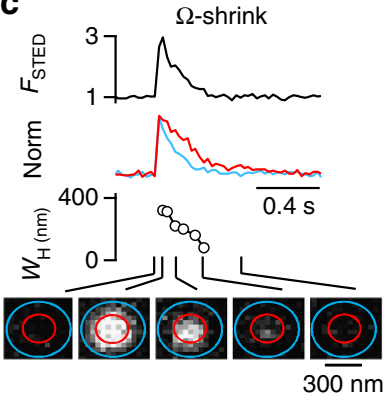

d

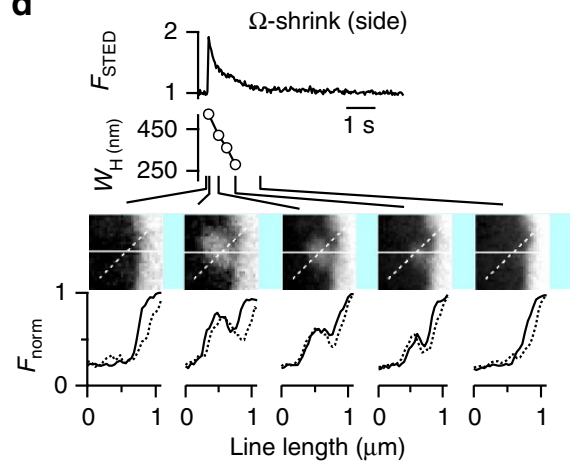

Figure 6 | $\Omega$-shrink fusion. (a) $\Omega$-shrink at confocal/A647/A488 setting. (b)Distribution of the $F_{647}$ decay $\tau$ during $\Omega$-shrink fusion at confocal/A647/ A488 setting ( 115 spots, 60 cells, data binned every $0.5 \mathrm{~s}$ ). (c,d) $\Omega$-shrink at STED/cell-bottom (c) and STED/cell-centre setting (d). In c, $F_{\text {STED_n }}$ in the inner circle (red) and the outer ring (blue) are also plotted to show spot shrinkage. (d) Left two images, average of two single images; right three images, average of eight single images.

changes was observed after stimulation. Thus, FC fusion was inexistent or rare. This suggestion was not due to limit in time resolution, because we imaged every $5-15 \mathrm{~ms}$, which detected nearly all NPY-EGFP releasing granules (for example, Fig. 1e). It was also not due to the stimulus we used $\left(\right.$ depol $\left._{1 \mathrm{~s}}\right)$, because FC fusion was absent with two other stimuli: a 2-ms depolarization train at $5-15 \mathrm{~Hz}$ for $30 \mathrm{~s}$ that mimicked an action potential train (48 spots, 5 cells), and high potassium application $(50 \mathrm{mM}, 53$ spots, 4 cells).

Spot size changes are not caused by movement. The spot enlargement or shrinking we observed was not due to focal plane changes, because while some spots enlarged or shrank, preexisting fluorescent structures $1-2 \mu \mathrm{m}$ away did not change (Supplementary Fig. 5a-c). Could it be due to localized $(<1-2 \mu \mathrm{m})$ membrane movement that pushes the $\Omega$-shaped membrane profile into the cytosol in the $\mathrm{z}$ axis? Four sets of evidence exclude this possibility.

First, the fluorescence within $\sim 0.5-1 \mu \mathrm{m}$ surrounding the spot remained unchanged at the STED cell-bottom setting $(n=101$ spots, for example, Supplementary Fig. 6), indicating no movement surrounding the spot. Similar results were observed using FM4-64 to label membrane (Supplementary Fig. 7a) ${ }^{28}$ and Atto 488 to identify fusion modes ( $n=75$ spots, 6 cells, Supplementary Fig. $7 b-d)$. Second, at the cell-centre setting where the movement to the cytosol could be resolved at the microscopic $x / y$ plane (illustrated in Supplementary Fig. 8c-e), we did not observe any such movements ( $n=45$ spots; for example, Supplementary Fig. $8 \mathrm{f}-\mathrm{j})$. Third, at a $\mathrm{z}$ resolution of $\sim 100-150 \mathrm{~nm}$ total internal reflection fluorescence microscopy (TIRF, cell-bottom setting, $30 \mu \mathrm{M}$ Alexa 555 in bath) showed that after $\operatorname{depol}_{1 \mathrm{~s}}$ the spot size may shrink completely ( $\Omega$-shrink, 33 out of 178 spots, Supplementary Fig. 9a), shrink partially (including $\Omega$-shrinkstay and $\Omega$-shrink-close; 33/178 spots, Supplementary Fig. 9b), enlarge (including $\Omega$-enlarge-stay and $\Omega$-enlarge-close; $17 / 178$ spots, Supplementary Fig. 9c), or remain unchanged (including $\Omega$-stay and $\Omega$-close; 95/178 spots, Supplementary Fig. 9d). This result excludes movements to the cytosol of $\sim 350 \mathrm{~nm}$ or larger (the confocal $\mathrm{z}$ resolution is $\pm \sim 350 \mathrm{~nm}$ ) as the cause for spot size changes. Fourth, to monitor such movements at STED cellbottom setting (A488 in bath), we switched the focal plane every $70 \mathrm{~ms}$ between the cell-bottom (control) and a focal plane that was $300 \mathrm{~nm}$ above (upper). Spots induced by depol $_{1 \mathrm{~s}}$ were brighter and focused at the control focal plane, but dimmer and out of focus at the upper focal plane (131 spots). When spots dimmed and shrank completely (28 spots, Supplementary Fig. 10a) or partially (40 spots, Supplementary Fig. 10b) at the control plane, $F_{\text {STED }}$ at the upper plane also decreased, but slightly faster (Supplementary Fig. 10a,b). Thus, spot shrinking at the control plane is not due to an upward movement towards the cytosol. Similarly, all spots (21 spots) that became brighter and larger at the control plane were also brighter at the upper plane (Supplementary Fig. 10c). In conclusion, the spot size change we observed is not due to movement to the cytosol, but to actual structural changes.

Seven modes observed with other image settings. The seven modes were not only observed with the A647/A488 setting, but also with other settings, which validates our observations. When we used only one dye (A647) and excited it weakly, the spot size could remain the same ( $n=42$ spots), increase ( $n=7$ spots), or decrease (to some extent or till undetectable, $n=23$ spots; Supplementary Fig. 11; 7 cells), indicating that the spot size changes were not due to photo-toxicity caused by strong excitation. When we excited A488 strongly, but A647 weakly, depol s $_{1}$ induced 62 spots $(9$ cells) showing six modes ( $\Omega$-stay, 9 spots; $\Omega$-close, 12 spots; $\Omega$-enlarge-stay, 6 spots; $\Omega$-enlarge-close, 0 spots; $\Omega$-shrink-stay, 9 spots; $\Omega$-shrink-close, 15 spots; $\Omega$-shrink, 11 spots; Supplementary Fig. 12). When we replaced A647 and A488 with Atto 655 (strong excitation/confocal) and Atto 488 (weak excitation/confocal), depol ${ }_{1 \mathrm{~s}}$ induced 143 spots (7 cells) showing seven modes ( $\Omega$-stay, 8 spots; $\Omega$-close, 45 spots; $\Omega$ enlarge-stay, 7 spots; $\Omega$-enlarge-close, 3 spots; $\Omega$-shrink-stay, 19 spots; $\Omega$-shrink-close, 31 spots; $\Omega$-shrink, 30 spots; Fig. $7 \mathrm{c}-\mathrm{i}$ ). With these settings, the percentage of each mode (obtained from smaller data sets) was roughly similar to those obtained with A647/A488 setting.

Three close modes mediate rapid and slow endocytosis. Three sets of evidence in A647/A488 experiments suggest that close fusion $(\Omega$-close, $\Omega$-shrink-close, $\Omega$-enlarge-close) mediates whole-cell rapid (a few seconds) and slow (tens of seconds) endocytosis. First, if we assigned an upstep at every spot's onset and a downstep at pore closing time ( $F_{647}$ decay onset) with an amplitude correction for $\Omega$-shrink-close and $\Omega$-enlarge-close (Fig. 8a, see Methods), the up and downstep interval in close events ranged from $0.3-30 \mathrm{~s}(n=312$; Fig. $8 \mathrm{~b})$, covering both rapid and slow endocytic time frame.

Second, summation of the up and downsteps from all spots (636 spots) yielded reconstructed net exo- and endocytosis 
a

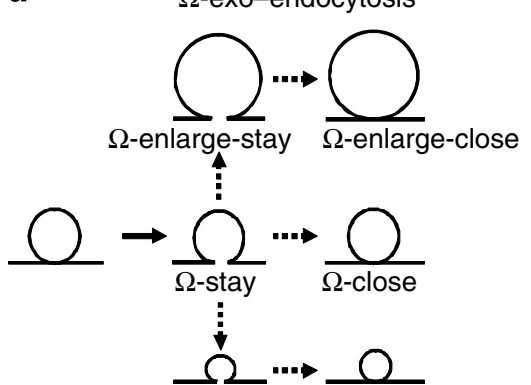

$\Omega$-shrink-stay $\Omega$-shrink-close

$$
\frac{\dot{\vdots}}{\Omega \text {-shrink }}
$$

d

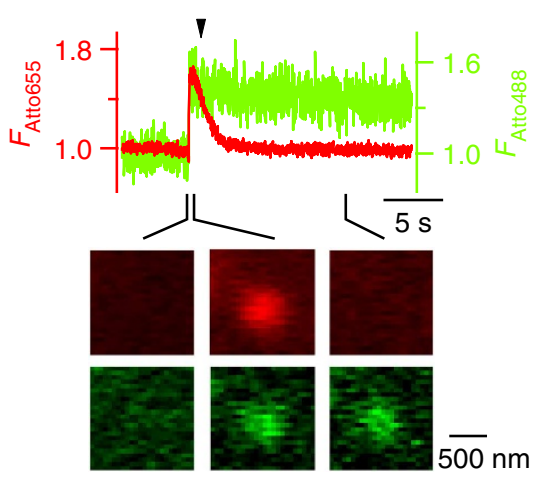

g

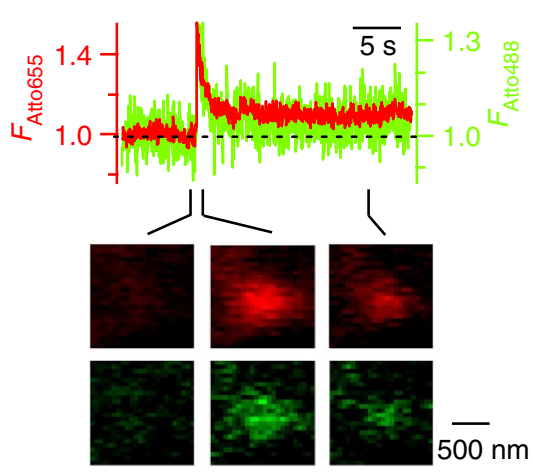

b

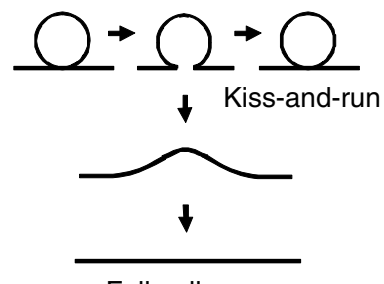

Full-collapse

Classical model e

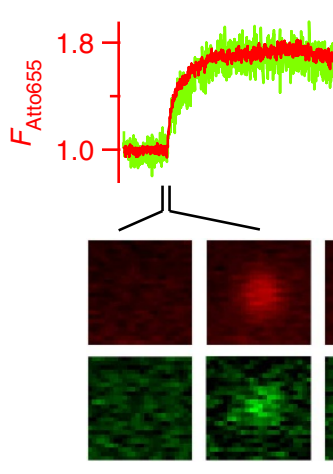

h

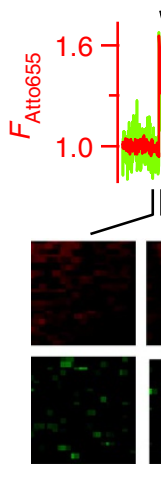

C

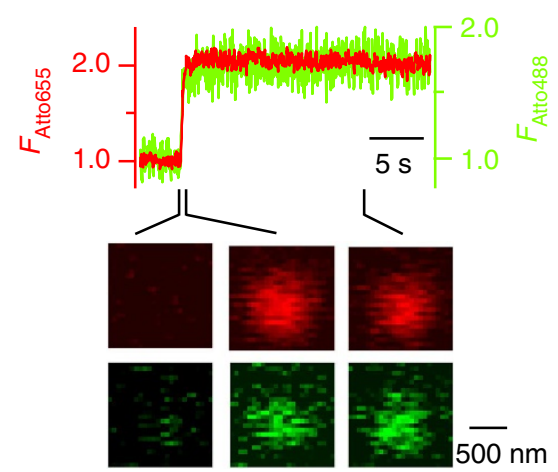

$\mathbf{f}$

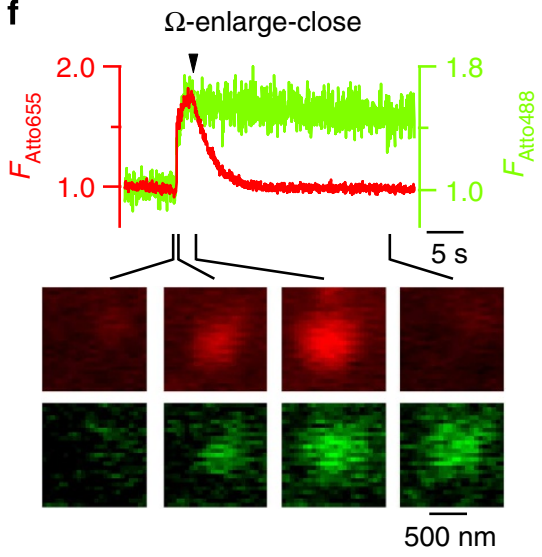

i

$\Omega$-shrink

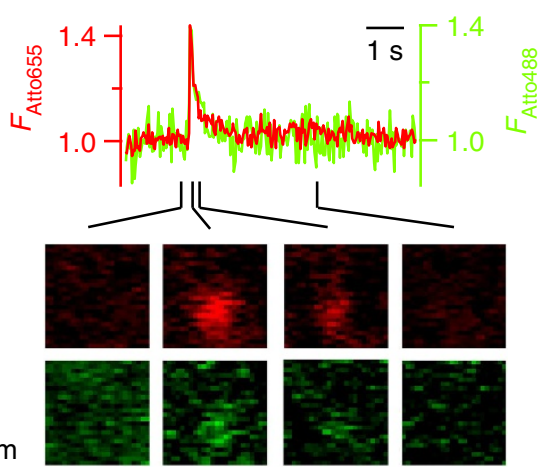

$5 \overline{00} \mathrm{~nm}$

Figure 7 | Seven fusion modes confirmed by imaging with Atto 655 and Atto 488. (a, b) Schematic drawings of our model called $\Omega$-exo-endocytosis (a, seven modes) and the classical FC and KR model (b). Dotted arrows mean that the transition may or may not take place. (c) Atto 655 fluorescence intensity $\left(F_{\text {Atto655, }}\right.$ red), Atto 488 fluorescence intensity $\left(F_{\text {Atto488, }}\right.$ green) and sampled images (average of 5-10 frames) at times indicated (lines) are plotted versus time for a spot undergoing $\Omega$-stay fusion. $F_{\text {Atto655 }}$ and $F_{\text {Atto } 488}$ were normalized to the mean value before spot appeared. Images were collected every 17-34 ms at the confocal cell-bottom setting with Atto 655 (strong excitation) and Atto 488 (weak excitation) in the bath. (d-i) Similar to c, but for spots undergoing $\Omega$-close (d), $\Omega$-enlarge-stay (e), $\Omega$-enlarge-close (f), $\Omega$-shrink-stay $(\mathbf{g}$ ), $\Omega$-shrink-close (h) and $\Omega$-shrink (i).

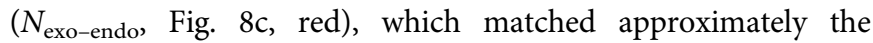
corresponding whole-cell endocytosis (Fig. 8c, black, 60 cells) in both time course and amplitude. The match was also observed when we divided cells into four groups based on capacitance decay: decay to baseline within $15 \mathrm{~s}$ (group 1), decay by $>80 \%$ in $30 \mathrm{~s}$ (group 2, not including group 1), decay by $30-80 \%$ in $30 \mathrm{~s}$ (group 3 ) and decay by $<30 \%$ in $30 \mathrm{~s}$ (group 4 , Fig. $8 \mathrm{~d}$ ). $N_{\text {exo-endo }}$ and capacitance decayed rapidly with a $\tau$ of $2.8 \mathrm{~s}$ and $3.9 \mathrm{~s}$, respectively, in group 1; but decayed slowly with a $\tau$ of 8.1 and $13.8 \mathrm{~s}$, respectively, in group 2 . In groups $3-4, N_{\text {exo-endo }}$ and capacitance changes were similar and did not return to baseline. The fraction that did not decay for $N_{\text {exo-endo }}$ and capacitance was similar in all groups (Fig. 8e). These results suggest that close modes mediate most whole-cell rapid and slow endocytosis.

Third, since prolonged whole-cell dialysis blocks endocyto$\operatorname{sis}^{21,29}$, we used this feature to determine whether close modes cause whole-cell endocytosis. In 4 cells, depol $_{1 \mathrm{~s}}$ induced wholecell endocytosis and 78\% (53/68 spots) close fusion within $1 \mathrm{~min}$ after break-in, but induced no whole-cell endocytosis, and only $3 \%$ close fusion ( $1 / 31$ spots, Fig. $8 f, n=4$ cells) 6 min later, suggesting that close modes cause whole-cell endocytosis. 
a

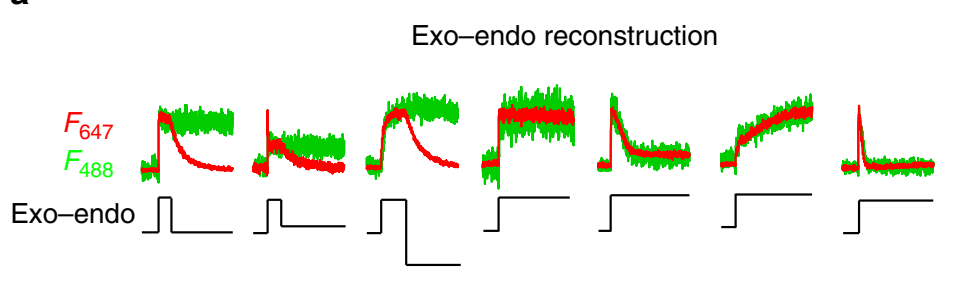

C

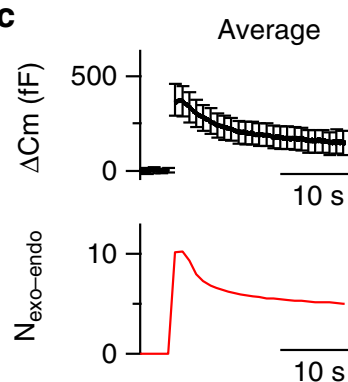

d
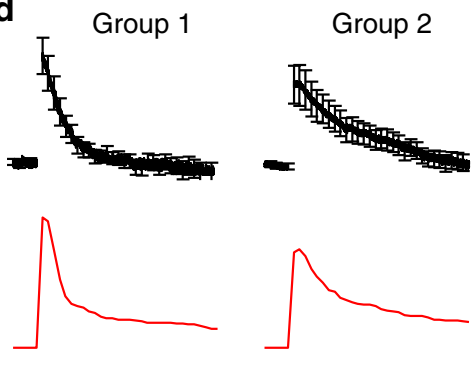
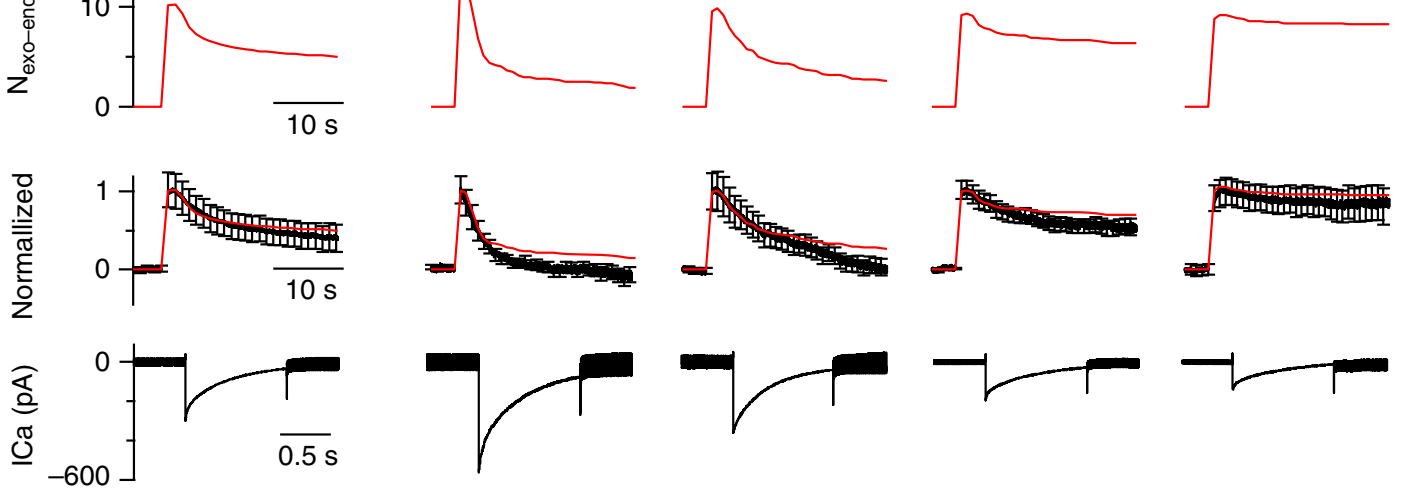

Group 4

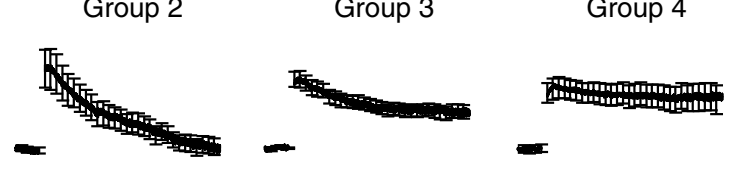

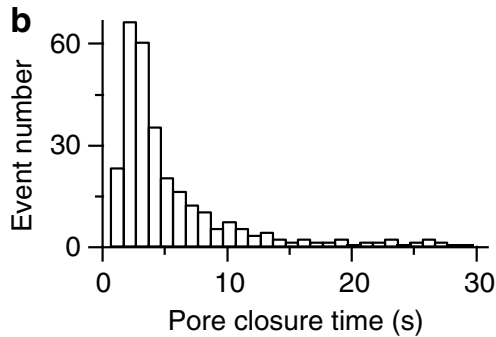

Pore closure time (s)

\section{0}
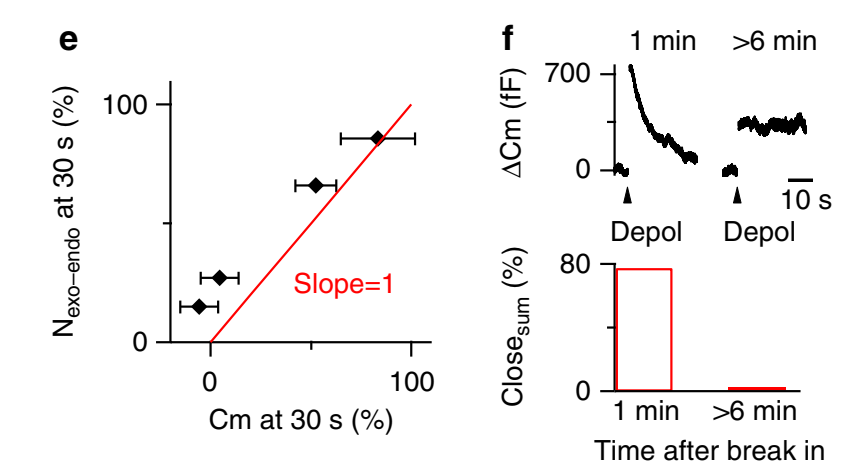

Figure 8 | Close modes mediate rapid and slow endocytosis of the cell. (a) Examples showing net exo- and endocytosis (lower: exo-endo, see also Methods) reconstructed from fluorescence changes (upper: $F_{647}, F_{488}$ ) during $\Omega$-close, $\Omega$-shrink-close, $\Omega$-enlarge-close, $\Omega$-stay, $\Omega$-shrink-stay, $\Omega$-enlargestay and $\Omega$-shrink fusion (left to right). (b) Pore closure time (from open to close, not from stimulation time to closure) distribution for three 'close' modes (312 spots). (c) The mean $\mathrm{Cm}$ ( \pm s.e.m., every $1 \mathrm{~s}$, baseline subtracted, upper), $N_{\text {exo-endo }}$ per cell (middle upper) and ICa (lower) induced by depol ${ }_{1 \mathrm{~s}}$ (to $+10 \mathrm{mV}$ ). $\mathrm{Cm}$ (black) and $N_{\text {exo-endo }}(\mathrm{red})$ traces are also normalized and superimposed for comparison (middle lower). Data were from 636 spots in 60 cells. (d) Similar to ce except that data in c were divided into four groups based on $\mathrm{Cm}$ decay: (1) decay to baseline in $15 \mathrm{~s}$ ( $165 \mathrm{spots}, 11$ cells), (2) decay by $>80 \%$ in 30 s except group 1 (101 spots, 10 cells), (3) decay by $30-80 \%$ in 30 s (173 spots, 18 cells) and (4) decay by <30\% in 30 s (197 spots, 21 cells). (e) The percentage of $N_{\text {exo-endo }}$ at $30 \mathrm{~s}$ after depol ${ }_{1 \mathrm{~s}}$ (compared with the peak $N_{\text {exo-endo }}$ ) plotted versus the corresponding undecayed $\mathrm{Cm}$ percentage at $30 \mathrm{~s}$ after stimulation from four groups described in $\mathbf{d}$ (left to right, group 1-4). A line (red) with a slope of 1 is also plotted. Error bars are s.e.m. The spot number and cell number are described in $\mathbf{d}$. (f) The mean $\mathrm{Cm}$ change (upper, $\Delta \mathrm{Cm}$ ) and the percentage of close fusion (Close sum lower, including $\Omega$-close, $\Omega$-shrink-close and $\Omega$-enlarge-close) induced by depol ${ }_{1 \mathrm{~s}}$ within $1 \mathrm{~min}$ (left, 53/68 spots are 'close' fusion) and $>6$ min (right, $1 / 31$ spots is close fusion) after whole-cell break-in from the same cell ( $n=4$ cells).

Calcium influx determines fusion modes. From group 4 to 1 , ICa increased from $175 \pm 25 \mathrm{pA}(n=21$ cells) to $576 \pm 61 \mathrm{pA}$ $(n=11$ cells, Fig. $8 \mathrm{~d})$, close events (three close modes) increased and became dominant, whereas the stay events (three stay modes) and $\Omega$-shrink decreased (Fig. 9a). The correlation between ICa and close fusion (Fig. 9a, red), between ICa and stay fusion (Fig. 9a blue), and between ICa and $\Omega$-shrink (Fig. 9a, black) are causal, because in cells showing large ICa $(>450 \mathrm{pA}$ during $<10 \mathrm{~ms}$ depolarization to $+10 \mathrm{mV}$ ), when we reduced ICa using a 1-s depolarization to $+50 \mathrm{mV}(102 \pm 8 \mathrm{pA}, n=12$ cells, Fig. 9b), close events were nearly fully blocked (3.1\%), whereas stay events and $\Omega$-shrink increased (Fig. 9c, open symbols, 12 cells, 64 spots). Thus, large ICa triggered close modes, whereas low ICa promoted stay modes and $\Omega$-shrink.

Dynamin mediates fusion pore closure. In control cells with an

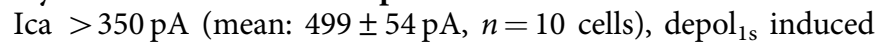


whole-cell endocytosis and 73\% (48/66 spots) of close fusion (Fig. 9d). With the dynamin inhibitor dynasore $(80 \mu \mathrm{M}, 20-$ $30 \mathrm{~min}$ ) in the bath in cells with an ICa $>350 \mathrm{pA}$ (mean:

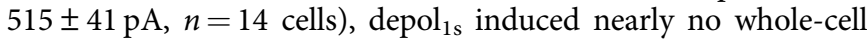
endocytosis ( $\sim 10-30 \%$ at $30 \mathrm{~s}$ after depol $_{1 \mathrm{~s}}$, Fig. $\left.9 \mathrm{~d}\right)$ and only $8 \%$ (5/63 spots) close fusion (Fig. 9d). Similarly, including in the pipette solution the dynamin inhibitory peptide (QVPSRPNRAP, $20 \mu \mathrm{M}$; Tocris $)^{30}$, which inhibits dynamin interaction with amphiphysin, largely blocked whole-cell endocytosis and reduced close fusion to $9 \%(4 / 45$ spots $)$ in cells with an ICa $>350 \mathrm{pA}(585 \pm 73 \mathrm{pA}, n=6)$. These results suggest that dynamin mediates fusion pore closure, which in turn mediates whole-cell rapid and slow endocytosis.

$\Omega$-profile retains vesicular membrane protein. To determine whether the $\Omega$-shaped membrane profile, which was observed in all seven modes, holds vesicular membrane proteins from diffusion, we imaged cells expressing a vesicular membrane protein VAMP2 tagged with EGFP (VAMP2-EGFP, weak excitation). A647 (bath, strong excitation) was included for identifying fusion modes (see Methods and Supplementary Fig. 13). Depol $1 \mathrm{~s}$ induced 66 A647 spots (14 cells) that coincided with VAMP2EGFP spots. VAMP2-EGFP fluorescence $\left(F_{\mathrm{VAMP} 2}\right)$ increased at A647 spot onset owing to the $\mathrm{pH}$ increase upon fusion (Fig. 10) ${ }^{31}$.

During $\Omega$-stay, $F_{\mathrm{VAMP} 2}$ and spot size remained stable $(n=12$ spots, Fig. 10a). During $\Omega$-close, spot size remained stable $(n=13$ spots, Fig. 10b). Pore closure lead to $F_{647}$ decay, whereas $F_{\text {VAMP2 }}$ decreased partially in $30 \mathrm{~s}$ ( $n=13$ spots, Fig. $10 \mathrm{~b})$, consistent with cavicapture that takes $100 \mathrm{~s}$ or longer for re-acidification ${ }^{15,31}$. During $\Omega$-enlarge-stay, $F_{\mathrm{VAMP} 2}$ remained stable, whereas VAMP2-EGFP spot size increased $(n=5$, Fig. 10c). During $\Omega$-shrink-stay, $F_{647}$ decrease (to $20 \pm 2 \%, n=9$ ) in the shrink phase was not accompanied by parallel $F_{\mathrm{VAMP} 2}$ decrease, but a delayed and smaller $F_{\mathrm{VAMP} 2}$ decrease (to $67 \pm 7 \%, n=9$, for example, Fig. 10d). However, VAMP2-EGFP spot $W_{\mathrm{H}}$ decreased in parallel with A647 spot $W_{\mathrm{H}}$ (for example, Fig. 10d, $n=9$ ), suggesting that the $\Omega$-shaped membrane prevents or slows down diffusion of vesicular membrane proteins.

For $\Omega$-shrink-close, A647 and VAMP2-EGFP spot size reduced in parallel (for example, Fig. 10e, $n=5$ ). $F_{\mathrm{VAMP} 2}$ did not decay during the shrink phase just like $\Omega$-shrink-stay, but decayed with a delay slowly $(n=5$, Fig. $10 \mathrm{e})$, likely due to slow reacidification $^{15,31}$. During $\Omega$-shrink, $F_{\mathrm{VAMP} 2}$ decreased to baseline $(n=22)$. If shrinking was rapid, a VAMP2-EGFP diffusion cloud was observed (Fig. 10f). If shrinking was slow, VAMP2-EGFP spot size reduced in parallel with the A647 spot size (Fig. 10g). $F_{\text {VAMP2 }}$ reduction significantly lagged behind $F_{647}$ reduction, reminiscent of the delay observed in $\Omega$-shrink-stay and $\Omega$-shrinkclose (Fig. 10d,e). Thus, in all seven modes, $\Omega$-shaped membrane profiles may prevent or slow down diffusion of vesicular membrane proteins.

At $\sim 20 \mathrm{~s}$ after the spot appeared, reducing the bath $\mathrm{pH}$ to 5.5 by applying MES solution decreased the $\mathrm{pH}$-sensitive $F_{\text {VAMP2 }}$ (ref. 31 ) to baseline for stay events (including $\Omega$-stay, $\Omega$-enlargestay and $\Omega$-shrink-stay, $n=9$ spots,Fig. $10 \mathrm{~h}$, left), but not for close events (including $\Omega$-close and $\Omega$-shrink-close, $n=8$ spots, Fig. 10, right), confirming the pore open or close status, as determined by A647 imaging.

\section{Discussion}

We establish a new exo-endocytosis model, termed $\Omega$-exoendocytosis (Fig. 7a), where the $\Omega$-profile fuison $_{\text {does not dilate, but }}$ changes in seven patterns through size transformation and fusion pore closure. This model is fundamentally different from the classical FC/KR model. FC is redefined as $\Omega$-shrink, which merges fused vesicles with the plasma membrane by shrinking, a

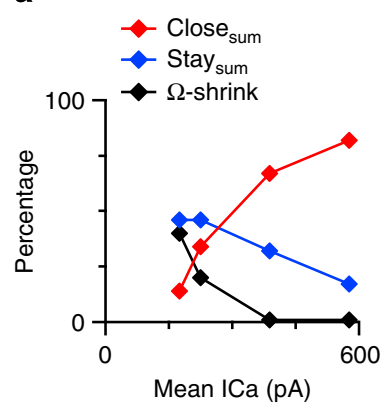

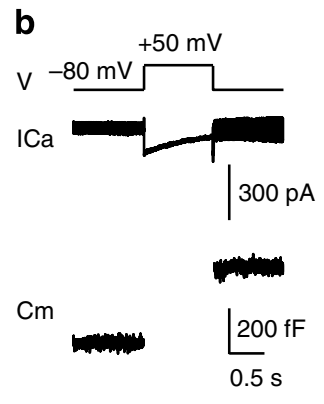

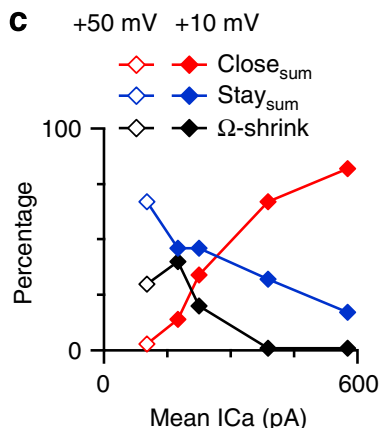

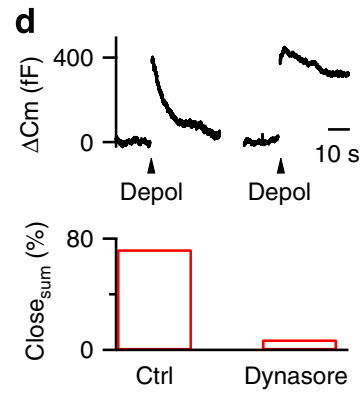

Figure 9 | Strong calcium influx triggers dynamin-dependent close fusion modes and low calcium promotes stay modes and $\mathbf{\Omega}$-shrink. (a) The percentage of Close $_{\text {sum }}$ (including all three close modes), Stay sum (including three stay modes) and $\Omega$-shrink plotted versus the mean ICa in four groups described in Fig. 8d (stimulation: depol ${ }_{1 \mathrm{~s}}$ to $+10 \mathrm{mV}$ ). The percentage was calculated within each group. (b) Sample ICa and $\mathrm{Cm}$ induced by a $1 \mathrm{~s}$ depolarization to $+50 \mathrm{mV}$. This cell showed an ICa of $\sim 500 \mathrm{pA}$ during a $10 \mathrm{~ms}$ depolarization to $+10 \mathrm{mV}$ (not shown). (c) Re-plotting a (solid symbols), but including data similar to those shown in $\mathbf{b}$ (open symbols), where $1 \mathrm{~s}$ depolarization to $+50 \mathrm{mV}$ induced the smallest ICa as compared with the

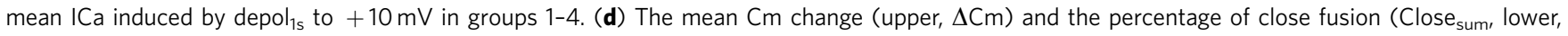
including $\Omega$-close, $\Omega$-shrink-close and $\Omega$-enlarge-close) induced by depol ${ }_{1 \mathrm{~s}}$ in control (10 cells, 66 spots, left) and in cells bathed with $80 \mu \mathrm{M}$ dynasore (14 cells, 63 spots, right). In both groups, cells with an ICa $>350$ pA were selected for analysis.

Figure 10 | $\Omega$-profile retains vesicle membrane protein VAMP2. (a-g) $F_{647}$ (red), $F_{V A M P 2}$ (green), $W_{H}$ of A647 (red) and VAMP2-EGFP (green) spot, and sampled A647 (red) and VAMP2-EGFP (green) images (at times indicated with lines) for spots undergoing $\Omega$-stay (a), $\Omega$-close (b), $\Omega$-enlargestay (c), $\Omega$-shrink-stay (d), $\Omega$-shrink-close (e) and $\Omega$-shrink (f: rapid shrinking, diffusion cloud; $\mathbf{g}$ : slow shrinking, size reduction observed). Cells were expressed with VAMP2-EGFP and stimulated by depol ${ }_{1 \mathrm{~s}}$ with $\mathrm{A} 647$ in the bath. $W_{\mathrm{H}}$ is not measured in $\mathbf{f}$, because VAMP2-EGFP rapidly diffused into a cloud, which did not reflect the $\Omega$-shaped membrane profile size. VAMP2-EGFP spots appeared slightly ( $\sim 50-100 \mathrm{~nm}$ in $\left.W_{H}\right)$ larger than corresponding A647 spots (for example, Fig. 10a,b), because VAMP2-EGFP was located at the membrane, whereas A647 was inside the $\Omega$-shaped structure. (h) The $F_{647}$ and $F_{\text {VAMP2 }}$ changes in response to a bath $\mathrm{pH}$ change from 7.4 to 5.5 (upper) for spots undergoing $\Omega$-stay (left) and $\Omega$-close (right). 
a

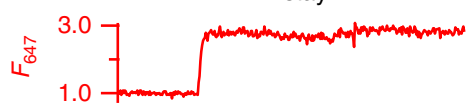

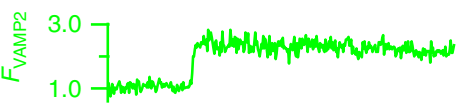

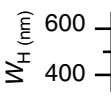

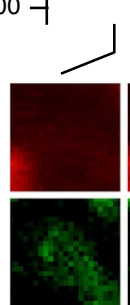

0
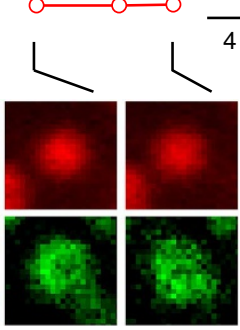

$5 \overline{00 \mathrm{~nm}}$
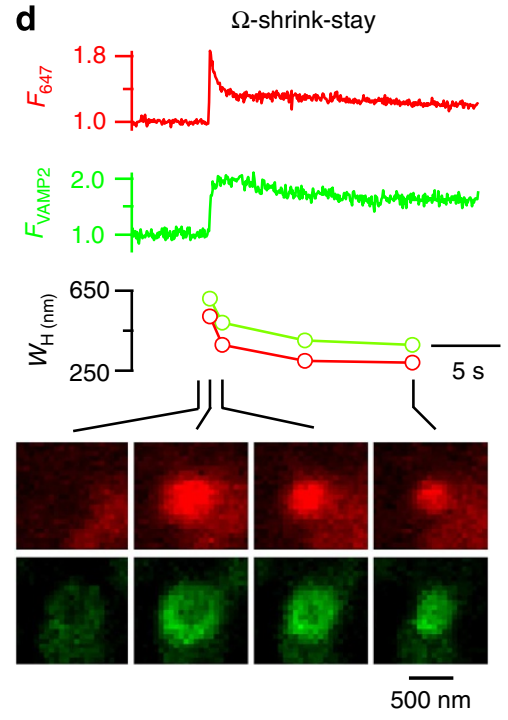

g
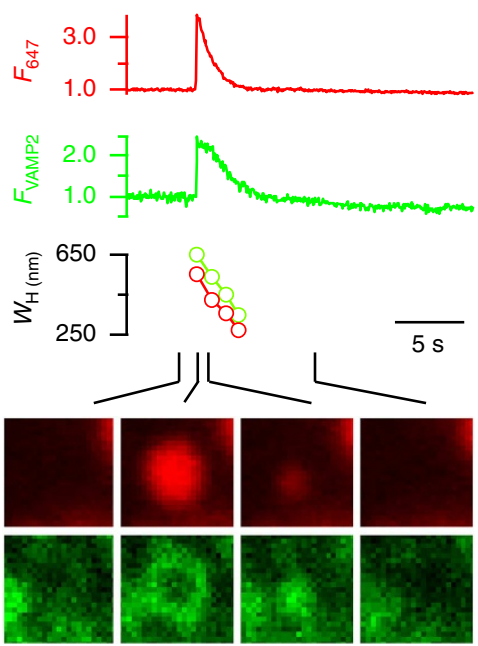

$5 \overline{00 \mathrm{~nm}}$ b
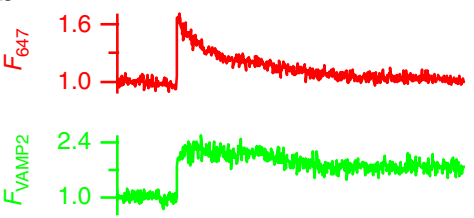

$\left.\begin{array}{ll}\stackrel{\widehat{E}}{\underline{E}} & 650 \\ S^{I} & \\ & 450\end{array}\right]$
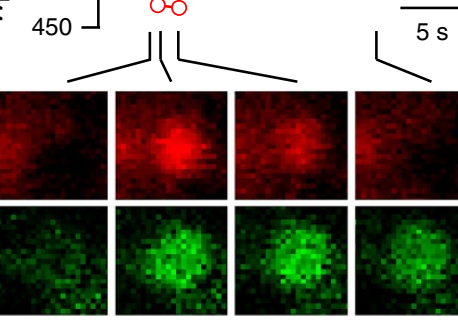

$5 \overline{00 \mathrm{~nm}}$
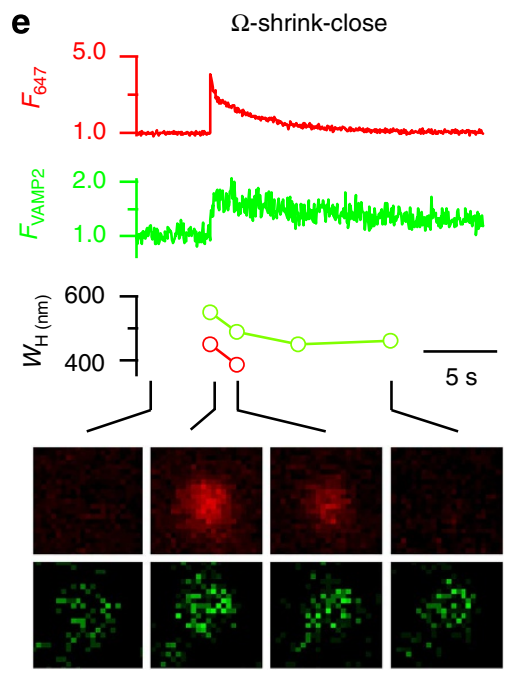

$\overline{500 \mathrm{~nm}}$
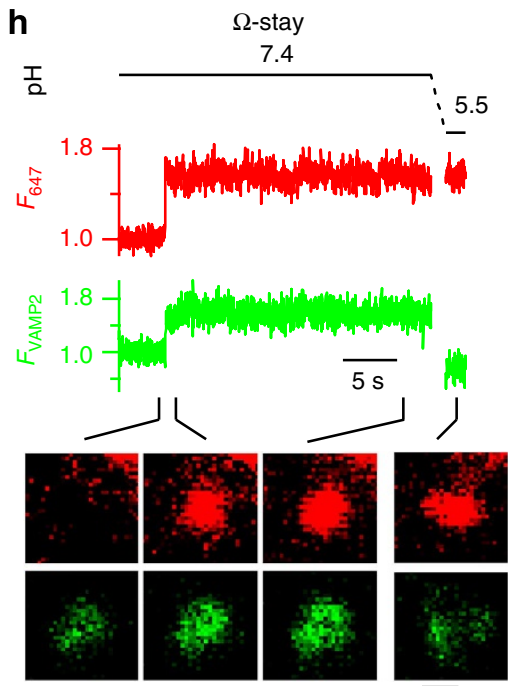

$50 \overline{\mathrm{nm}}$
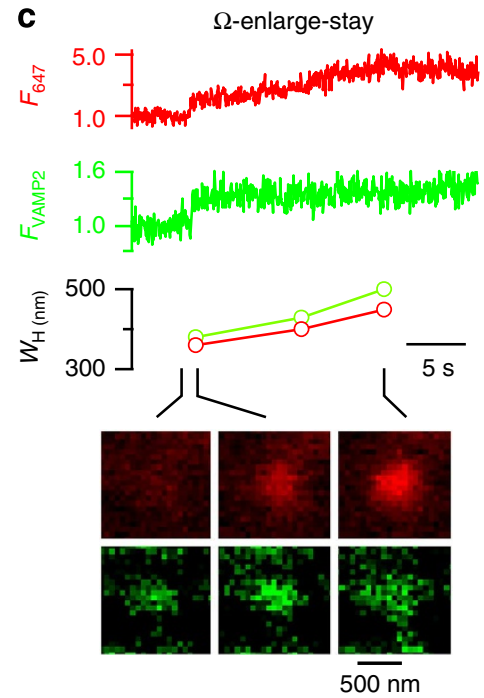

f

$\Omega$-shrink (rapid)
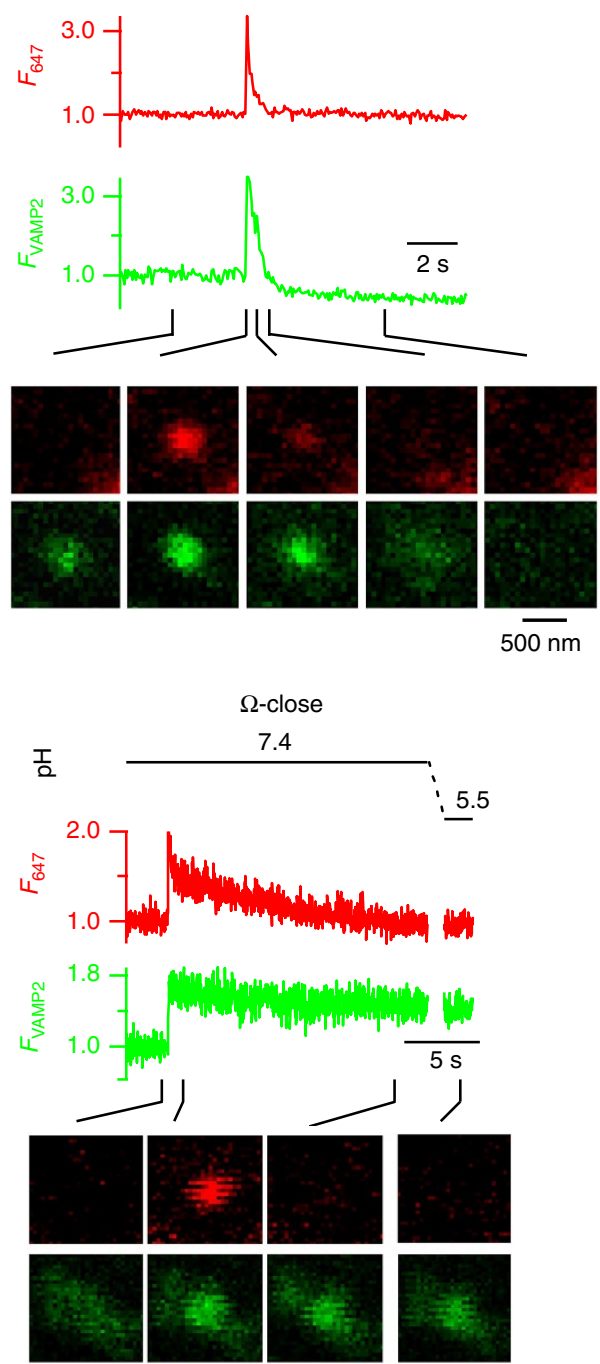

$50 \overline{\mathrm{nm}}$

but not dilating the $\Omega$-shaped membrane profile. KR is redefined as close fusion, including $\Omega$-close, $\Omega$-shrink-close and $\Omega$-enlargeclose, which may generate different-sized vesicles. Close fusion is triggered by strong calcium influx and requires dynamin to close the pore. Close fusion is the dominant mechanism mediating whole-cell rapid and slow endocytosis. It may also regulate vesicle size by shrinking or enlarging the $\Omega$-shaped membrane profile before fusion pore closure. Consequently, the enlargement 
( $\Omega$-enlarge-close) may mediate bulk endocytosis, a form of endocytosis defined as formation of large vesicles from the plasma membrane $e^{5,6}$. While strong calcium influx triggers close fusion, weak calcium influx facilitates stay fusion $(\Omega$-stay, $\Omega$-shrink-stay, $\Omega$-enlarge-stay) and $\Omega$-shrink. Unlike the irreversible FC model, transition between the different modes in the $\Omega$-exo-endocytosis model is flexible (Fig. 7a). Finally, most vesicular membrane proteins are maintained in the $\Omega$-shaped membrane during various modes of fusion by a mechanism that needs further investigation.

Our model may account for most observations previously interpreted with FC/KR model. For example, $\Omega$-shrink not only accounts for the merging of fused vesicles with the plasma membrane previously attributed to FC, but also allows for the flexibility to close the fusion pore anytime during shrinking (Fig. 7a). The pore of $\Omega$-shrink fusion is much larger than $4 \mathrm{~nm}$, because $\Omega$-shrink released the $\sim 4 \mathrm{~nm}$ NPY-EGFP ${ }^{32}$ rapidly with a $\tau$ of $33 \pm 4 \mathrm{~ms}(n=49$ spots, for example, Fig. 1e). $\Omega$-shrink with a large pore may explain all live-cell data previously interpreted as FC, such as rapid content release, fusion pore conductance increase above a detection limit (corresponds to $\sim 3-5 \mathrm{~nm}$ pore), and disappearance of fusion-generated vesicular images in endocrine cells and neurons $13,15,25,27,31,33-37$.

Could $\Omega$-shrink replace FC at synapses? FC was first suggested by Heuser and Reese $^{2}$ based on freeze-fracture electron microscopic observation that there are more large openings (diameter: $60-120 \mathrm{~nm}$ ) at $50 \mathrm{~ms}$ than at 3-5 ms after stimulation at neuromuscular junctions ${ }^{38}$. It predicts that as pore dilates, large openings become dominant. However, large openings are not dominant at any time measured ${ }^{2}$. Using similar techniques and preparation, Ceccarelli et al. ${ }^{7}$ questioned whether FC exists $^{39,40}$. A recent study shows a widened neck of an $\Omega$ profile consistent with the FC model ${ }^{41}$. However, FC has not been observed in live cells. An increased surface area ${ }^{42}$ and release of $\sim 15 \mathrm{~nm}$ quantum $\operatorname{dot}^{43}$ at live retinal and hippocampal nerve terminals support FC, but can also be interpreted with $\Omega$-shrink that has a large pore. Further work is needed to confirm $\Omega$-shrink fusion in live synapses.

By comparing endocytosis reconstructed from 636 fusion events with concurrently measured whole-cell endocytosis, we found that fusion pore closure during close fusion mediates most slow endocytosis within $30 \mathrm{~s}$ after stimulation (Fig. 8c,d, group 2-4). We cannot exclude classical endocytosis beyond $30 \mathrm{~s}$, although closure of stay fusion may also contribute to whole-cell endocytosis. Our finding challenges the traditional view that slow endocytosis is mediated by classical endocytosis.

Can close fusion contribute to slow endocytosis at synapses? Inhibition of slow endocytosis by blocking clathrin-dependent endocytosis ${ }^{5}$ and the existence of a readily retrievable pool ${ }^{44-46}$ argue against this possibility. However, it remains unclear whether close fusion is clathrin-dependent and whether stay fusion generates the readily retrievable pool. Further study is needed to determine whether close fusion contributes to slow endocytosis at synapses.

Rapid endocytosis in endocrine cells and neurons is hypothesized to be caused by $\mathrm{KR}^{8,19,47}$, rapid classical endocytosis ${ }^{46,48}$ or bulk endocytosis ${ }^{42,49}$. Which mechanism mediates rapid endocytosis remains unresolved, because each endocytic mode's contribution had not been reconstructed for comparison with whole-cell endocytosis. Providing such comparison for the first time, we found that close fusion (three close modes), which includes bulk endocytosis (mediated by $\Omega$-enlarge-close), underlies rapid endocytosis (Fig. 8d, group 1).

The three close modes provide a mechanism to regulate vesicle size. Hence, they may contribute to or cause vesicle size variation observed within a cell and among different cells ${ }^{50,51}$. Since vesicle size is proportional to quantal size $e^{50,51}$, regulation of three close modes may in turn modulate quantal size, which defines exocytosis strength, such as increased vesicle size and synaptic strength observed after animal activity ${ }^{52}$. Previous studies suggest that low calcium concentration triggers rapid $\mathrm{KR}^{17,18}$, which predicts rapid whole-cell endocytosis when calcium influx is reduced or buffered. In contrast, reducing or buffering calcium influx abolishes endocytosis in chromaffin cells and neurons ${ }^{20,22-24,53,54}$. The present work may explain this discrepancy, because in studies suggesting that KR is triggered by low calcium, an indirectly detected $\Omega$-profile, but not pore closure is interpreted as $\mathrm{KR}^{17,18}$. Such an interpretation is analogous to low calcium-facilitated stay modes reported here (Fig. 9). We found that strong calcium influx triggers close fusion to mediate whole-cell endocytosis, consistent with the finding that calcium influx triggers whole-cell endocytosis (Figs 8,9) 20-24,53,54.

Our finding that strong calcium influx triggers close fusion is apparently consistent with results showing that $\mathrm{KR}$ is dominant at $90 \mathrm{mM}$ extracellular calcium ${ }^{14}$. However, increasing extracellular calcium $(50 \mathrm{mM})$ decreases ICa during depolarization ${ }^{15}$, implying that prolonged extracellular, but not intracellular calcium increase facilitates KR. Consistent with this implication, prolonged intracellular calcium increase does not promote $\mathrm{KR}^{55}$. Taken together, we conclude that strong calcium influx induced by transient depolarization triggers close fusion.

Rapid KR (within seconds) is proposed as a simple reversal of fusion pore opening without dynamin involvement, whereas cavicapure (slow KR) may require dynamin ${ }^{4,56,57}$. The present work provides experimental data showing that not only slow, but also rapid fusion pore closure is mediated by dynamin (Fig. 9d). Our finding that close fusion is a dominant endocytic mechanism seems in contrast to the infrequent KR observed in cell-attached recordings. This difference is likely due to different definitions and recording conditions. In cell-attached recordings, $\mathrm{KR}$ is detected as equal sized capacitance up- and down-steps that occur within $2 \mathrm{~s}^{7,35,37,58,59}$, which would exclude $\Omega$-shrink-close, $\Omega$-enlarge-close and $\Omega$-close that closes after $2 \mathrm{~s}$. Furthermore, close modes were triggered by strong calcium influx during transient depolarization (Fig. 8), whereas KR in cell-attached recordings is often detected at rest or with high potassium depoarization $^{13,14,35,37,59}$, which does not promote close fusion.

How the granule dense core copes with structural changes during $\Omega$-exo-endocytosis is unknown. It might be squeezed out or dissolved first and then released during $\Omega$-shrink ${ }^{60,61}$, but might stay as observed in pituitary lactotrophs ${ }^{61}$ during stay or close fusion.

In summary, the $\Omega$-exo-endocytosis model may explain most live-cell data previously interpreted with $\mathrm{FC} / \mathrm{KR}$ model. It may apply to large vesicles in many other cell types, such as pancreatic cells, adipocytes, blood cells, glial cells and neurons that secret dopamine, peptides and hormones ${ }^{62}$. Whether it applies to small synaptic vesicles deserves consideration, because neuroendocrine chromaffin cells and nerve terminals are similar in many aspects that were traditionally interpreted with classical exo- and endocytosis models, such as capacitance upsteps and flickers, rapid and slow content release, calcium-triggered rapid and slow endocytosis, bulk endocytosis and proteins involved in exo- and endocytosis $1,4,5,10,11,20,23,24,63$.

Vesicle fusion has been imaged by many techniques, including TIRF $^{31}$ or polarized TIRF imaging ${ }^{64}$, two-photon imaging with extracellular dye that our technique is based on ${ }^{25}$, and interference reflection microscopy ${ }^{42}$. Using confocal and STED microscopy, we achieved the highest spatial and temporal resolution currently available, $\sim 90 \mathrm{~nm} / 5-15 \mathrm{~ms}$. We detected pore closure by differential excitation of two dyes at a temporal 
resolution $(\sim 0.3 \mathrm{~s})$ much faster than other imaging methods ${ }^{31}$. Without relying on protein overexpression, our method is much more efficient in capturing vesicle fusion (Fig. 1e,f). Our imaging method opens the door for studying the mechanisms that generate and regulate the $\Omega$-profile, or more generally, membrane curvature.

\section{Methods}

Primary bovine chromaffin cell culture. We prepared primary chromaffin cell culture as described previously ${ }^{65}$. In brief, fresh adult (21-27 months old) bovine adrenal glands (local abattoir), were immersed in pre-chilled $1 \times$ Lock's buffer on ice containing: $\mathrm{NaCl}, 145 \mathrm{mM} ; \mathrm{KCl}, 5.4 \mathrm{mM} ; \mathrm{Na}_{2} \mathrm{HPO} 4,2.2 \mathrm{mM} ; \mathrm{NaH}_{2} \mathrm{PO}$, $0.9 \mathrm{mM}$; glucose, $5.6 \mathrm{mM}$; HEPES, $10 \mathrm{mM}$; pH 7.3 adjusted with $\mathrm{NaOH}$. Glands were perfused with $1 \times$ Lock's buffer, then infused with Lock's buffer containing collagenase $\mathrm{P}\left(1.5 \mathrm{mg} \mathrm{ml}^{-1}\right.$, Roche), trypsin inhibitor $\left(0.325 \mathrm{mg} \mathrm{ml}^{-1}\right.$, Sigma $)$ and bovine serum albumin $\left(5 \mathrm{mg} \mathrm{ml}^{-1}\right.$, Sigma) and incubated at $37^{\circ} \mathrm{C}$ for $20 \mathrm{~min}$. The digested medulla was minced in Lock's buffer and filtered through a nylon mesh. The filtrate was centrifuged $(39 g, 4 \mathrm{~min})$, re-suspended in Lock's buffer and re-centrifuged until the supernatant was clear. Final cell pellet was re-suspended in pre-warmed DMEM low-glucose medium (Gibco) supplemented with $10 \%$ fetal bovine serum (Gibco) and plated onto poly-L-lysine $(0.005 \%$ w/v, Sigma) and laminin $\left(4 \mu \mathrm{g} \mathrm{ml}{ }^{-1}\right.$, Sigma)-coated glass coverslips. The cells were incubated at $37^{\circ} \mathrm{C}$ with $8 \% \mathrm{CO}_{2}$ and used within 1 week. Before plating, some cells were transfected by electroporation ( $2 \mu \mathrm{g}$ plasmid DNA containing NPY-EGFP or VAMP2-EGFP) using Basic Neuron Nucleofector Kit (Lonza, Program O-005).

Electrophysiology. At room temperature $\left(22-24^{\circ} \mathrm{C}\right)$, whole-cell voltage clamp and capacitance recordings were performed with an EPC-10 amplifier together with the software lock-in amplifier (PULSE, HEKA, Lambrecht, Germany) ${ }^{23,66}$. The holding potential was $-80 \mathrm{mV}$. The frequency of the sinusoidal stimulus was $1,000-1,500 \mathrm{~Hz}$ with a peak-to-peak voltage $\leq 50 \mathrm{mV}$. The bath solution contained $125 \mathrm{mM} \mathrm{NaCl}, 10 \mathrm{mM}$ glucose, $10 \mathrm{mM}$ HEPES, $5 \mathrm{mM} \mathrm{CaCl}_{2}, 1 \mathrm{mM} \mathrm{MgCl}_{2}, 4.5 \mathrm{mM}$ $\mathrm{KCl}, 0.001 \mathrm{mM}$ TTX and $20 \mathrm{mM}$ TEA, pH 7.3 adjusted with $\mathrm{NaOH}$. The pipette (3-6 M $\Omega$ ) solution contained $130 \mathrm{mM}$ Cs-glutamate, $0.5 \mathrm{mM}$ Cs-EGTA, $12 \mathrm{mM}$ $\mathrm{NaCl}, 30 \mathrm{mM}$ HEPES, $1 \mathrm{mM} \mathrm{MgCl} 2,2 \mathrm{mM}$ ATP and $0.5 \mathrm{mM}$ GTP, pH 7.2 adjusted with $\mathrm{CsOH}$. These solutions pharmacologically isolated calcium currents.

Imaging. With an inverted confocal microscope (TCS SP5II, Leica, Germany, $100 \times$ oil objective, numerical aperture: 1.4), A647 (30 $\mu \mathrm{M}$ in bath, Invitrogen) and A488 (30-60 $\mu \mathrm{M}$ in bath, Invitrogen) were excited by a HeNe laser at $633 \mathrm{~nm}$ (maximum power: $20 \mathrm{~mW}$ ) and an Argon laser at $488 \mathrm{~nm}$ (maximum power: $25 \mathrm{~mW}$ ), respectively. Unless mentioned otherwise, the $633 \mathrm{~nm}$ laser was set at $60 \%$ of the maximum power, whereas $488 \mathrm{~nm}$ laser was set at $1.5-2 \%$. A647 fluorescence was collected with a photomultiplier at $643-700 \mathrm{~nm}$, whereas A488, with a GaAsP hybrid detection system at $498-580 \mathrm{~nm}$. The quantum efficiency of the hybrid detection system is two times higher than the photomultiplier, which improved the signal-to-noise ratio for A488 imaging. Both excitation and fluorescence collection were done simultaneously.

The inverted STED microscope has a resolution of $80 \mathrm{~nm}$ (TCS STED, Leica, Germany), which $(\sim 80-90 \mathrm{~nm})$ was confirmed with fluorescent bead measurements. A488 $(60 \mu \mathrm{M})$ was excited with an Argon laser at $488 \mathrm{~nm}$ at $20 \%$ of the maximum power (maximum power: $25 \mathrm{~mW}$ ), and depleted with a continuous wave fiber laser at $592 \mathrm{~nm}$ using the maximum power $(1.5 \mathrm{~W})$. The fluorescence was acquired by GaAsP hybrid detection system at $498-580 \mathrm{~nm}$. At $20 \%$ of the maximum power, $488 \mathrm{~nm}$ laser caused A488 bleaching after pore closure with a time course similar to that of A647 under the confocal setting.

Confocal or STED imaging area was $\sim 70-160 \mu \mathrm{m}^{2}$. Images were collected every $5-15 \mathrm{~ms}$ at $50-70 \mathrm{~nm}$ per pixel at the confocal/A647/A488 setting, every $26 \mathrm{~ms}$ at $40 \mathrm{~nm}$ per pixel at the STED/cell-bottom setting and every $36 \mathrm{~ms}$ at $40 \mathrm{~nm}$ per pixel at the STED/cell-centre setting.

For imaging at STED/cell-centre setting, we chose a focal plane that showed a clear edge between the solution and the cytosolic compartment of the cell, an indication that the cell membrane was approximately in parallel with the microscope's $\mathrm{z}$ axis. More specifically, at such a focal plane, the distance that covered $20-80 \%$ fluorescence changes between the solution and the cytosolic compartment was less than $300 \mathrm{~nm}$, beyond which the cell was not used. Furthermore, to clearly resolve the $\Omega$-profile or the FC profile, we only analysed fluorescent spots with an initial $W_{\mathrm{H}}$ more than $350 \mathrm{~nm}$. This selection avoided challenging our limited spatial resolution $(\sim 90 \mathrm{~nm})$ when a small spot shrank until undetectable.

TIRF imaging (Olympus FV1000) was taken every $100-200$ ms with a $60 \times$ oil immersion objective (NA: 1.45) and an EMCCD camera (Hamamatsu Photonics). Alexa 555 in the bath solution was excited by a HeNe laser at $543 \mathrm{~nm}$.

Fluorescence measurements and presentations. Images were analysed using ImageJ. The fluorescence intensity $\left(F_{647}, F_{488}, F_{\text {STED }}\right)$ from an area covering the fluorescence spot was measured at every image frame. For images shown in figures and movies, 2-4 frames were averaged at the confocal/A647/A488 setting, 2-3 frames were averaged at STED/cell-bottom setting and 2-8 frames were averaged at STED/cell-centre setting. The averaging improved signals and usually did not sacrifice the time resolution, because most changes were much slower than the time needed for averaging (Figs 3-6). However, a fraction of 'shrink' fusion ( $\Omega$-shrink, $\Omega$-shrink-stay, $\Omega$-shrink-close) may take only $50-200 \mathrm{~ms}$ during the shrinking phase, in which we used 1-2 frames for averaging. $W_{\mathrm{H}}$ was measured from intensity profiles of 1-4 lines (for example, Fig. 2a,b) across the spot centre at $45^{\circ}$ or $90^{\circ}$ apart. At STED/cell-centre setting, $W_{\mathrm{H}}$ was measured from line profiles approximately in parallel with the cell membrane (Figs $3 c, 4 b, 5 c$,f and 6d). For line profiles, we normalized the peak fluorescence to 1 . For $F_{647}, F_{488}$ and $F_{\text {STED }}$, the value before the spot appeared was normalized to 1 .

Identifying fusion modes with a single dye. When A647 (strong excitation) and EGFP were imaged (Fig. 10), most fusion modes except $\Omega$-shrink and $\Omega$-close, could be readily distinguished from $F_{647}$ change alone, as shown in Figs 3-6. Although $F_{647}$ decayed in both $\Omega$-shrink and $\Omega$-close fusion, the decay $\tau$ for $\Omega$ shrink (1.09 $\pm 0.11 \mathrm{~s}, n=115$ spots, Supplementary Fig. 13a) was much faster than that of $\Omega$-close ( $2.9 \pm 0.1 \mathrm{~s}, n=210$ spots, Supplementary Fig. 13b). Since the decay, $\tau$ distribution for $\Omega$-shrink overlapped little with that of $\Omega$-close (Supplementary Fig. 13c), we classified spots with a decay $\tau<1.7 \mathrm{~s}$ as $\Omega$-shrink. This criterion excluded $\Omega$-close, because the decay $\tau$ for all $\Omega$-close events was $>1.7 \mathrm{~s}$ (Supplementary Fig. 13). Spots with a decay $\tau>2 \mathrm{~s}$ were classified as $\Omega$-close, in which $8 \%$ could be due to $\Omega$-shrink owing to the overlap distribution of the decay $\tau$ (Supplementary Fig. 13c). Such a small error should not significantly affect our main conclusion.

With a single dye (A488, strong excitation) in STED imaging, we identified the fusion modes based on changes in $F_{\mathrm{STED}}$ and $W_{\mathrm{H}}$ similar to confocal imaging. STED imaging was more sensitive in detecting the $W_{\mathrm{H}}$ change, making it easier to distinguish between $\Omega$-shrink and $\Omega$-close.

Exo- and endocytosis reconstruction. In confocal/A647/A488 experiments, we labelled each spot at the spot onset as an upstep with an amplitude of 1, and a downstep only for three 'close' modes at the pore closure time, that is, the onset of A647 bleaching while A488 remained unchanged (Fig. 8a). The downstep amplitude was corrected for $\Omega$-shrink-close and $\Omega$-enlarge-close by raising the relative fluorescence changes during the 'shrink or 'enlarge' phase to a power of $2 / 3$, which was approximately proportional to the granule surface area or capacitance. This correction was based on our observation that the fluorescence change was approximately proportional to the $W_{\mathrm{H}}$ change raised to a power of 3 . Here we did not consider granule size differences for each fusion mode, because the mean spot $W_{\mathrm{H}}$ at the fusion onset was similar for seven fusion modes.

Measuring the pore closure time. For close modes, the onset of the $F_{647}$ decay when $F_{488}$ remained unchanged was taken as pore closure time. The $F_{647}$ decay time constant was $2.9 \pm 0.1 \mathrm{~s}(n=210)$, meaning that at $0.3 \mathrm{~s}, F_{647}$ decays by $10 \%$, which could be well resolved (for example, arrows in Figs $3 \mathrm{~d}-\mathrm{f}, 4 \mathrm{c}$ and $5 \mathrm{~d}-\mathrm{f}$ ). Thus, our time resolution for pore closure was $0.3 \mathrm{~s}$. If pore closure was less than our resolution, we assigned an arbitrary value of $0.3 \mathrm{~s}$.

Data selection for analysis. The data within the first 2 min after whole-cell breakin were used to avoid whole-cell endocytosis rundown (see also Fig. $8 \mathrm{f}^{21}$. Cells showing prominent endocytosis overshoot were discarded, because we focused on determining whether 'close' modes are responsible for compensatory endocytosis, and the overshoot often curtailed the capacitance increase to a negative value, making it difficult to compare whole-cell exo-endo with reconstructed exoendocytosis.

Statistics. The statistical test used is $t$-test or paired $t$-test. The data were expressed as mean \pm s.e.m.

\section{References}

1. Sudhof, T. C. The synaptic vesicle cycle. Annu. Rev. Neurosci. 27, 509-547 (2004).

2. Heuser, J. E. \& Reese, T. S. Structural changes after transmitter release at the frog neuromuscular junction. J. Cell Biol. 88, 564-580 (1981).

3. Miller, T. M. \& Heuser, J. E. Endocytosis of synaptic vesicle membrane at the frog neuromuscular junction. J. Cell Biol. 98, 685-698 (1984).

4. Alabi, A. A. \& Tsien, R. W. Perspectives on kiss-and-run: role in exocytosis, endocytosis, and neurotransmission. Annu. Rev. Physiol. 75, 393-422 (2013).

5. Saheki, Y. \& De Camilli, P. Synaptic vesicle endocytosis. Cold Spring Harb. Perspect. Biol. 4, a005645 (2012).

6. Wu, L. G., Hamid, E., Shin, W. \& Chiang, H. C. Exocytosis and endocytosis: modes, function, and coupling mechanisms. Annu. Rev. Physiol. 76, 301-331 (2014).

7. Ceccarelli, B., Hurlbut, W. P. \& Mauro, A. Turnover of transmitter and synaptic vesicles at the frog neuromuscular junction. J. Cell Biol. 57, 499-524 (1973). 
8. Fesce, R., Grohovaz, F., Valtorta, F. \& Meldolesi, J. Neurotransmitter release, fusion or 'kiss and run'? Trends Cell Biol. 4, 1-4 (1994).

9. Hell, S. W. Far-field optical nanoscopy. Science 316, 1153-1158 (2007).

10. Lindau, M. \& Alvarez de Toledo, G. The fusion pore. Biochim. Biophys. Acta 164, 167-173 (2003).

11. Jackson, M. B. \& Chapman, E. R. The fusion pores of $\mathrm{Ca}(2+)$-triggered exocytosis. Nat. Struct. Mol. Biol. 15, 684-689 (2008).

12. Neher, E. \& Marty, A. Discrete changes of cell membrane capacitance observed under conditions of enhanced secretion in bovine adrenal chromaffin cells. Proc. Natl Acad. Sci. USA 79, 6712-6716 (1982).

13. Albillos, A. et al. The exocytotic event in chromaffin cells revealed by patch amperometry. Nature 389, 509-512 (1997).

14. Ales, E. et al. High calcium concentrations shift the mode of exocytosis to the kiss-and-run mechanism. Nat. Cell Biol. 1, 40-44 (1999).

15. Perrais, D., Kleppe, I. C., Taraska, J. W. \& Almers, W. Recapture after exocytosis causes differential retention of protein in granules of bovine chromaffin cells. J. Physiol. 560, 413-428 (2004).

16. He, L. \& Wu, L. G. The debate on the kiss-and-run fusion at synapses. Trends Neurosci. 30, 447-455 (2007).

17. Fulop, T. \& Smith, C. Physiological stimulation regulates the exocytic mode through calcium activation of protein kinase $\mathrm{C}$ in mouse chromaffin cells. Biochem. J. 399, 111-119 (2006).

18. Fulop, T., Radabaugh, S. \& Smith, C. Activity-dependent differential transmitter release in mouse adrenal chromaffin cells. J. Neurosci. 25, 7324-7332 (2005).

19. Elhamdani, A., Azizi, F. \& Artalejo, C. R. Double patch clamp reveals that transient fusion (kiss-and-run) is a major mechanism of secretion in calf adrenal chromaffin cells: high calcium shifts the mechanism from kiss-and-run to complete fusion. J. Neurosci. 26, 3030-3036 (2006)

20. Artalejo, C. R., Henley, J. R., McNiven, M. A. \& Palfrey, H. C. Rapic endocytosis coupled to exocytosis in adrenal chromaffin cells involves $\mathrm{Ca}^{2+}, \mathrm{GTP}$, and dynamin but not clathrin. Proc. Natl Acad. Sci. USA 92, 8328-8332 (1995).

21. Smith, C. \& Neher, E. Multiple forms of endocytosis in bovine adrenal chromaffin cells. J. Cell Biol. 139, 885-894 (1997).

22. Sankaranarayanan, S. \& Ryan, T. A. Calcium accelerates endocytosis of vSNAREs at hippocampal synapses. Nat. Neurosci. 4, 129-136 (2001).

23. $\mathrm{Wu}, \mathrm{X}$. S. et al. $\mathrm{Ca}(2+)$ and calmodulin initiate all forms of endocytosis during depolarization at a nerve terminal. Nat. Neurosci. 12, 1003-1010 (2009).

24. Hosoi, N., Holt, M. \& Sakaba, T. Calcium dependence of exo- and endocytotic coupling at a glutamatergic synapse. Neuron 63, 216-229 (2009).

25. Takahashi, N., Kishimoto, T., Nemoto, T., Kadowaki, T. \& Kasai, H. Fusion pore dynamics and insulin granule exocytosis in the pancreatic islet. Science 297, 1349-1352 (2002).

26. Engisch, K. L. \& Nowycky, M. C. Compensatory and excess retrieval: two types of endocytosis following single step depolarizations in bovine adrenal chromaffin cells. J. Physiol. 506 Pt 3 591-608 (1998).

27. He, L. et al. Compound vesicle fusion increases quantal size and potentiates synaptic transmission. Nature 459, 93-97 (2009).

28. Hoopmann, P., Rizzoli, S. O. \& Betz, W. J. Imaging synaptic vesicle recycling by staining and destaining vesicles with FM dyes. Cold Spring Harb. Protoc. 2012, $77-83$ (2012).

29. Renden, R. \& Von Gersdorff, H. Synaptic vesicle endocytosis at a CNS nerve terminal: faster kinetics at physiological temperatures and increased endocytotic capacity during maturation. J. Neurophysiol. 98, 3349-3359 (2007).

30. Grabs, D. et al. The SH3 domain of amphiphysin binds the proline-rich domain of dynamin at a single site that defines a new $\mathrm{SH} 3$ binding consensus sequence. J. Biol. Chem. 272, 13419-13425 (1997).

31. Taraska, J. W., Perrais, D., Ohara-Imaizumi, M., Nagamatsu, S. \& Almers, W. Secretory granules are recaptured largely intact after stimulated exocytosis in cultured endocrine cells. Proc. Natl Acad. Sci. USA 100, 2070-2075 (2003).

32. Palm, G. J. et al. The structural basis for spectral variations in green fluorescent protein. Nat. Struct. Biol. 4, 361-365 (1997).

33. Chow, R. H., von Ruden, L. \& Neher, E. Delay in vesicle fusion revealed by electrochemical monitoring of single secretory events in adrenal chromaffin cells. Nature 356, 60-63 (1992).

34. Wang, C. T. et al. Different domains of synaptotagmin control the choice between kiss-and-run and full fusion. Nature 424, 943-947 (2003).

35. Klyachko, V. A. \& Jackson, M. B. Capacitance steps and fusion pores of small and large-dense-core vesicles in nerve terminals. Nature 418, 89-92 (2002).

36. Nemoto, T. et al. Sequential-replenishment mechanism of exocytosis in pancreatic acini. Nat. Cell Biol. 3, 253-258 (2001).

37. He, L., Wu, X. S., Mohan, R. \& Wu, L. G. Two modes of fusion pore opening revealed by cell-attached recordings at a synapse. Nature 444, 102-105 (2006).

38. Heuser, J. E. Review of electron microscopic evidence favouring vesicle exocytosis as the structural basis for quantal release during synaptic transmission. Q. J. Exp. Physiol. 74, 1051-1069 (1989).
39. Torri-Tarelli, F., Grohovaz, F., Fesce, R. \& Ceccarelli, B. Temporal coincidence between synaptic vesicle fusion and quantal secretion of acetylcholine. J. Cell Biol. 101, 1386-1399 (1985).

40. Valtorta, F. et al. Neurotransmitter release and synaptic vesicle recycling. Neuroscience 35, 477-489 (1990).

41. Watanabe, S. et al. Ultrafast endocytosis at Caenorhabditis elegans neuromuscular junctions. Elife 2, e00723 (2013).

42. Llobet, A., Beaumont, V. \& Lagnado, L. Real-time measurement of exocytosis and endocytosis using interference of light. Neuron 40, 1075-1086 (2003).

43. Zhang, Q., Li, Y. \& Tsien, R. W. The dynamic control of kiss-and-run and vesicular reuse probed with single nanoparticles. Science 323, 1448-1453 (2009).

44. Fernandez-Alfonso, T., Kwan, R. \& Ryan, T. A. Synaptic vesicles interchange their membrane proteins with a large surface reservoir during recycling. Neuron 51, 179-186 (2006).

45. Wienisch, M. \& Klingauf, J. Vesicular proteins exocytosed and subsequently retrieved by compensatory endocytosis are nonidentical. Nat. Neurosci. 9, 1019-1027 (2006).

46. Hua, Y. et al. A readily retrievable pool of synaptic vesicles. Nat. Neurosci. 14, 833-839 (2011)

47. Von Gersdorff, H. \& Matthews, G. Dynamics of synaptic vesicle fusion and membrane retrieval in synaptic terminals. Nature 367, 735-739 (1994).

48. Balaji, J. \& Ryan, T. A. Single-vesicle imaging reveals that synaptic vesicle exocytosis and endocytosis are coupled by a single stochastic mode. Proc. Natl Acad. Sci. USA 104, 20576-20581 (2007).

49. Wu, W. \& Wu, L. G. Rapid bulk endocytosis and its kinetics of fission pore closure at a central synapse. Proc. Natl Acad. Sci. USA 104, 10234-10239 (2007).

50. Liu, G. Presynaptic control of quantal size: kinetic mechanisms and implications for synaptic transmission and plasticity. Curr. Opin. Neurobiol. 13, 324-331 (2003).

51. Lisman, J. E., Raghavachari, S. \& Tsien, R. W. The sequence of events that underlie quantal transmission at central glutamatergic synapses. Nat. Rev. Neurosci. 8, 597-609 (2007).

52. Steinert, J. R. et al. Experience-dependent formation and recruitment of large vesicles from reserve pool. Neuron 50, 723-733 (2006).

53. Wu, W., Xu, J., Wu, X. S. \& Wu, L. G. Activity-dependent acceleration of endocytosis at a central synapse. J. Neurosci. 25, 11676-11683 (2005)

54. Yamashita, T., Eguchi, K., Saitoh, N., Von Gersdorff, H. \& Takahashi, T. Developmental shift to a mechanism of synaptic vesicle endocytosis requiring nanodomain $\mathrm{Ca} 2+$. Nat. Neurosci. 13, 838-844 (2010).

55. Dernick, G., Alvarez de Toledo, G. \& Lindau, M. Exocytosis of single chromaffin granules in cell-free inside-out membrane patches. Nat. Cell Biol. 5, 358-362 (2003).

56. Graham, M. E., O’Callaghan, D. W., McMahon, H. T. \& Burgoyne, R. D. Dynamin-dependent and dynamin-independent processes contribute to the regulation of single vesicle release kinetics and quantal size. Proc. Natl Acad. Sci. USA 99, 7124-7129 (2002).

57. Holroyd, P., Lang, T., Wenzel, D., De, C. P. \& Jahn, R. Imaging direct, dynamin dependent recapture of fusing secretory granules on plasma membrane lawns from PC12 cells. Proc. Natl Acad. Sci. USA 99, 16806-16811 (2002).

58. Fernandez, J. M., Neher, E. \& Gomperts, B. D. Capacitance measurements reveal stepwise fusion events in degranulating mast cells. Nature 312, 453-455 (1984).

59. Alvarez de Toledo, G., Fernandez-Chacon, R. \& Fernandez, J. M. Release of secretory products during transient vesicle fusion. Nature 363, 554-558 (1993).

60. Monck, J. R., Oberhauser, A. F., Alvarez de Toledo, G. \& Fernandez, J. M. Is swelling of the secretory granule matrix the force that dilates the exocytotic fusion pore? Biophys. J. 59, 39-47 (1991)

61. Angleson, J. K., Cochilla, A. J., Kilic, G., Nussinovitch, I. \& Betz, W. J. Regulation of dense core release from neuroendocrine cells revealed by imaging single exocytic events. Nat. Neurosci. 2, 440-446 (1999).

62. Kasai, H., Takahashi, N. \& Tokumaru, H. Distinct initial SNARE configurations underlying the diversity of exocytosis. Physiol. Rev. 92, 1915-1964 (2012)

63. Wu, L. G., Ryan, T. A. \& Lagnado, L. Modes of vesicle retrieval at ribbon synapses, calyx-type synapses, and small central synapses. J. Neurosci. 27, 11793-11802 (2007).

64. Anantharam, A., Onoa, B., Edwards, R. H., Holz, R. W. \& Axelrod, D. Localized topological changes of the plasma membrane upon exocytosis visualized by polarized TIRFM. J. Cell Biol. 188, 415-428 (2010).

65. O'Connor, D. T. et al. Primary culture of bovine chromaffin cells. Nat. Protoc 2, 1248-1253 (2007)

66. Lindau, M. \& Neher, E. Patch-clamp techniques for time-resolved capacitance measurements in single cells. Pflugers Arch 411, 137-146 (1988).

\section{Acknowledgements}

We thank Dr. Fujun Luo for comments on the manuscript. We thank Drs. Carolyn Smith and Lei Xue for technical assistance in imaging. We thank Drs. Justin Taraska and 
Ronald Holz for providing NPY-EGFP and VAMP2-EGFP plasmids, respectively. This work was supported by the National Institute of Neurological Disorders and Stroke Intramural Research Program.

\section{Author contributions}

H.-C.C. and W.S. did most of the experiments, designed the experiments and participated in the writing. W.-D.Z., E.H., J.S., M.B. and P.J.W. did some experiments. A.J. and F.M. were involved in setting up optical systems and initial chromaffin cell cultures. L.-G.W. designed the experiments and wrote the manuscript.

\section{Additional information}

Supplementary Information accompanies this paper at http://www.nature.com/ naturecommunications

Competing financial interests: The authors declare no competing financial interests.

Reprints and permission information is available online at http://npg.nature.com/ reprintsandpermissions/

How to cite this article: Chiang, H.-C. et al. Post-fusion structural changes and their roles in exocytosis and endocytosis of dense-core vesicles. Nat. Commun. 5:3356 doi: $10.1038 /$ ncomms4356 (2014). 


\section{Erratum: Post-fusion structural changes and their roles in exocytosis and endocytosis of dense-core vesicles}

Hsueh-Cheng Chiang, Wonchul Shin, Wei-Dong Zhao, Edaeni Hamid, Jiansong Sheng, Maryna Baydyuk, Peter J. Wen, Albert Jin, Fanny Momboisse \& Ling-Gang Wu

Nature Communications 5:3356 doi: 10.1038/ncomms4356 (2014); Published 24 Feb 2014; Updated 27 Jan 2015

The right-hand side of Supplementary Fig. 13 was inadvertently deleted in the published Supplementary Information file for this Article. The correct version of the figure appears below. 

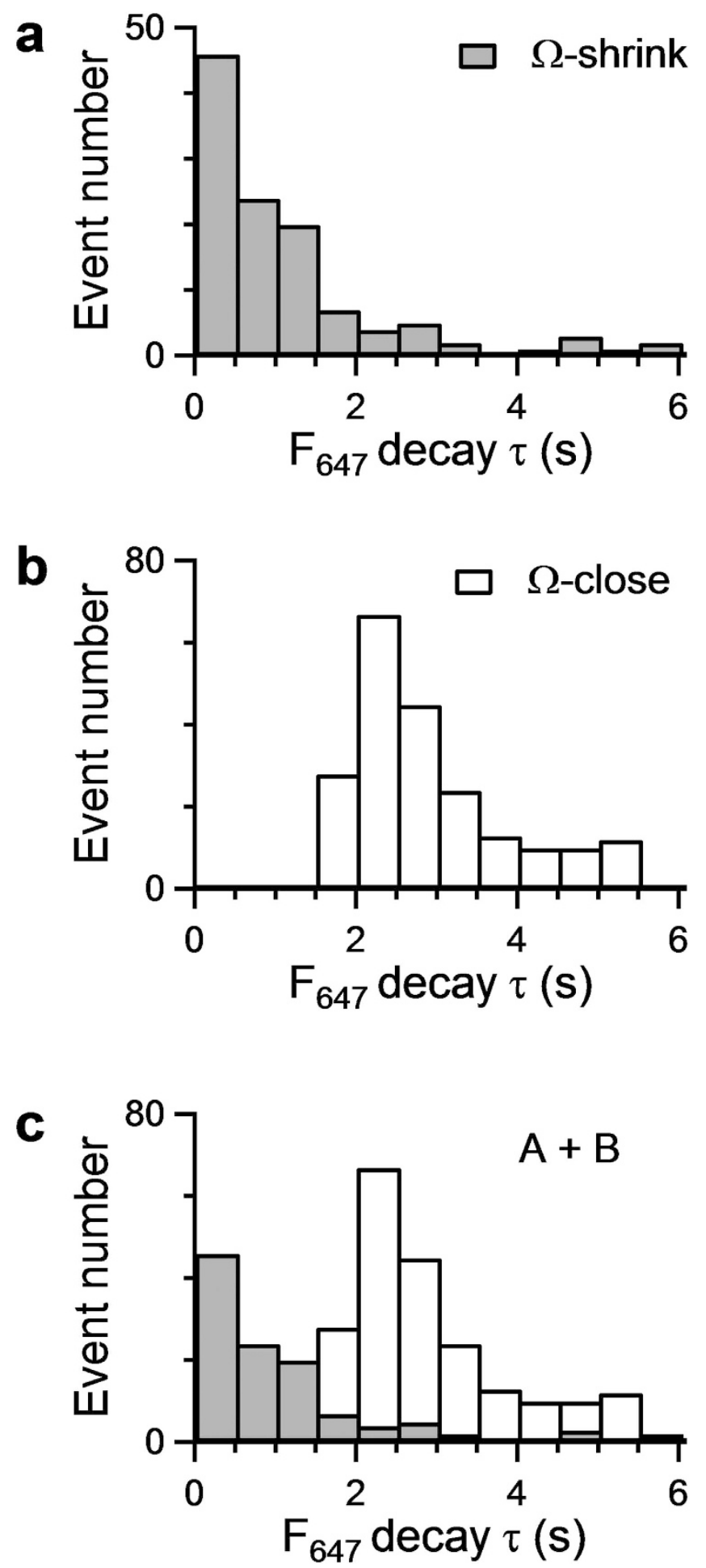